\title{
Ships and Offshore Structures
}

\section{Numerical Simulations of Vortex-Induced Vibrations on Vertical Cylindrical Structure with Different Aspect Ratio \\ --Manuscript Draft--}

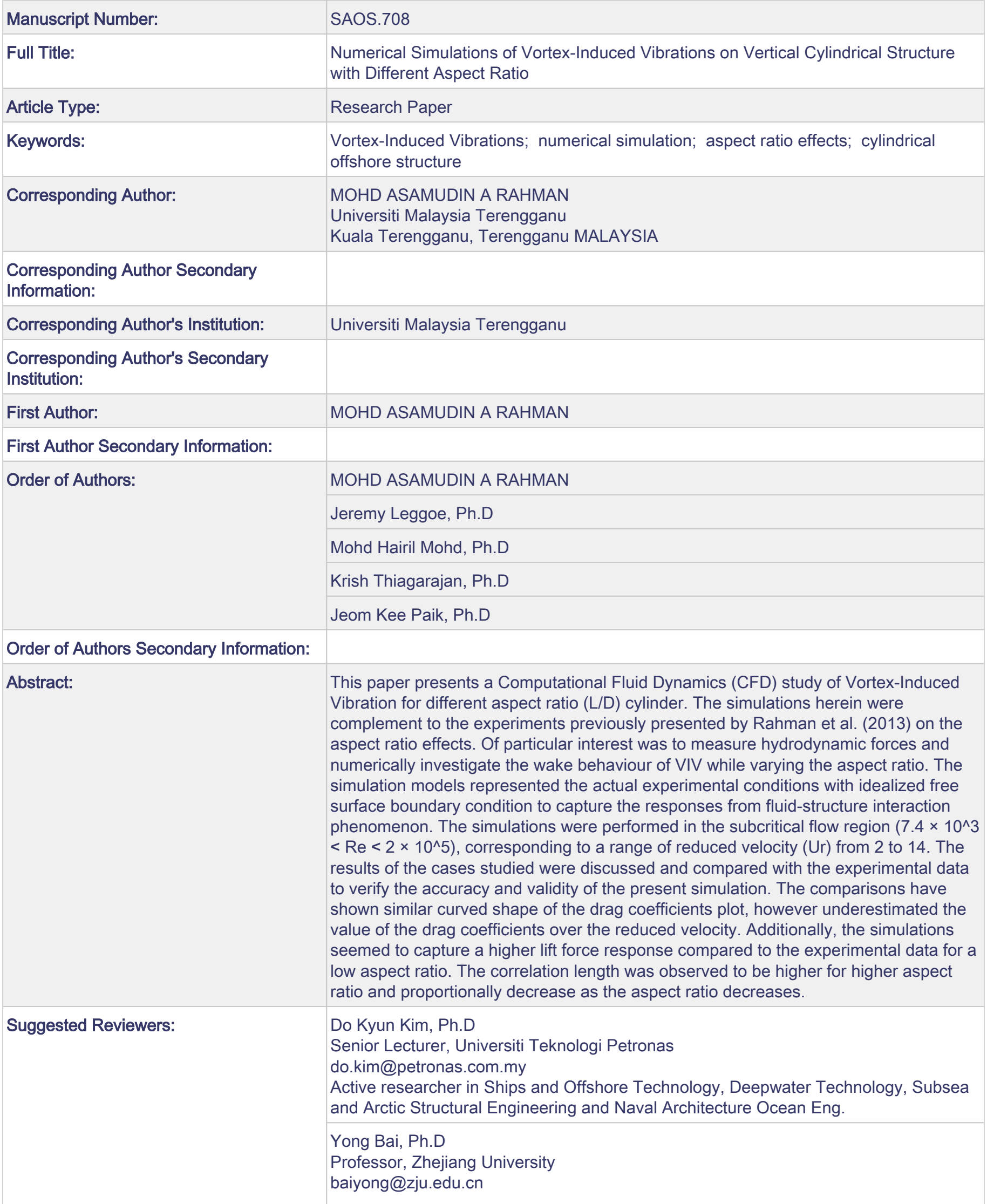


Scholar in:

1. Offshore pipeline and riser

2. Ocean engineering structure

3. Project risk analysis and safety evaluation

4. Composite Material tube

5. Offshore oil underwater production system

Mohammed Jameel, Ph.D

Senior Lecturer, University of Malaya

jameel@um.edu.my

Expert researcher in Structural Engineering (Nonlinear Dynamics, Finite Element

Analysis, Structural Reliability, Offshore structures, Fatigue Analysis,Tall structures and Wind \& Seismic analysis of buildings). 


\section{Numerical Simulations of Vortex-Induced Vibrations on Vertical Cylindrical Structure with Different Aspect Ratio}

Mohd Asamudin A Rahman ${ }^{\text {a, b* }}$, Jeremy Leggoe ${ }^{a}$, Mohd Hairil Mohd ${ }^{\text {, d }}$, Krish

Thiagarajan $^{\mathrm{c}}$ and Jeom Kee Paik ${ }^{\mathrm{d}}$

${ }^{a}$ School of Mechanical and Chemical Engineering, University of Western Australia, Crawley, WA, Australia

${ }^{b}$ School of Ocean Engineering, Universiti Malaysia Terengganu, Terengganu, Malaysia

${ }^{c}$ Department of Mechanical Engineering, University of Maine, Orono, USA

${ }^{d}$ The Ship and Offshore Research Institute, The Lloyd's Register Educational Trust

Research Centre of Excellence, Pusan National University, Busan, Republic of Korea

*Corresponding author. Email: mohdasamudin@umt.edu.my 


\title{
Numerical Simulations of Vortex-Induced Vibrations on Vertical Cylindrical Structure with Different Aspect Ratio
}

\author{
This paper presents a Computational Fluid Dynamics (CFD) study of Vortex- \\ Induced Vibration for different aspect ratio $(L / D)$ cylinder. The simulations \\ herein were complement to the experiments previously presented by Rahman et \\ al. (2013) on the aspect ratio effects. Of particular interest was to measure \\ hydrodynamic forces and numerically investigate the wake behaviour of VIV \\ while varying the aspect ratio. The simulation models represented the actual \\ experimental conditions with idealized free surface boundary condition to capture \\ the responses from fluid-structure interaction phenomenon. The simulations were \\ performed in the subcritical flow region $\left(7.4 \times 10^{3}<R e<2 \times 10^{5}\right)$, \\ corresponding to a range of reduced velocity $\left(U_{r}\right)$ from 2 to 14 . The results of the \\ cases studied were discussed and compared with the experimental data to verify \\ the accuracy and validity of the present simulation. The comparisons have shown \\ similar curved shape of the drag coefficients plot, however underestimated the \\ value of the drag coefficients over the reduced velocity. Additionally, the \\ simulations seemed to capture a higher lift force response compared to the \\ experimental data for a low aspect ratio. The correlation length was observed to \\ be higher for higher aspect ratio and proportionally decrease as the aspect ratio \\ decreases.
}

Keywords: Vortex-Induced Vibrations; numerical simulation; aspect ratio effects; cylindrical offshore structure

\section{Introduction}

VIV is a fluid-structure interaction phenomenon which occurs when a vibration of the structure is induced by forces from the vortices shed off the structure. It can occur in many practical situations, including fixed offshore structures, risers, mooring lines, floating structures, stacks, aircrafts, bridges, pipelines, engines, and tall structures. VIV is a multidisciplinary area encompassing continuum mechanics of fluids, structural mechanics, hydrodynamics, wavelet, and computational fluid mechanics; making it a 
complicated yet interesting area to investigate (Sarpkaya 2004). The interaction of fluids and structure can produce a potentially destructive force on the structure itself.

The flow of a uniform current around a circular cylinder has been extensively reviewed by Bearman (1984), Pantazopolous (1994), Sarpkaya (2004), Williamson and Govardhan (2008) and also in published books by Chen (1987), Blevins (1990) and Sumer and Fredsoe (1997). The behaviour of VIV phenomenon is normally governed by dimensional and non-dimensional parameters that control the oscillations of the cylinder. Among the parameters are the Reynolds number $(R e)$, density of fluid $(\rho)$, added mass $\left(m_{a}\right)$, fluid dynamic viscosity $(\mu)$, cylinder's diameter $(D)$, ambient velocity $(U)$, spring constant $(k)$ and structural damping ratio $\left(\zeta_{\mathrm{s}}\right)$ of the system.

Contributions towards understanding VIV are not only restricted to physical experiments, but also include Computational Fluid Dynamics (CFD) simulations to study VIV problems. Wilden et al. (2001), Thiagarajan et al. (2005), Kamarudin and Thiagarajan (2010), and Rosetti et al. (2013) are among the latest contributors, to name a few. With the availability of the various platforms of CFD software, simulation of flow past a cylinder has been extended to a 3D model. The capability of the CFD model to capture VIV behaviour enables the designer or researcher to predict the forces on the risers or any other offshore structures. For example, Blackburn and Karniadakis (1993) modelled a circular cylinder to investigate the interaction between the cylinder and the wake in free and forced vibration VIV. The authors have shown that simulations for both free and forced vibration have been able to produce most of the phenomena observed during a VIV experiment. In addition, Computational Fluid Dynamics (CFD) has become one of the important tools to study VIV at higher Reynolds numbers. Oakley et al. (2005), Halkyard et al. (2006), and Holmes (2008) are among the contributors to the numerical modelling for risers and spars experiencing VIV at higher 
Reynolds number. The Reynolds number in the CFD models scale was in the range of 8 $\times 10^{4}<\operatorname{Re}<2.5 \times 10^{5}$. However, the investigation of the correlation between the model scale and full scale of the Reynolds number is still questionable and requires further studies.

Most experiments and CFD simulations reported have focused on VIV experienced by an infinite length riser. For example, a 3D simulation of a riser was performed by Holmes et al. (2006) for a riser with $L / D=1400$, using the finite element methods. To the author's knowledge, there are still fewer study focussed on the low aspect ratio offshore structure in water. The influence of the aspect ratio on VIV response around a circular cylinder has been experimentally investigated and reported by Rahman et al. (2013), which is our interest in this paper. For some latest work along this line, see Goncalves et al. (2010, 2014) and Rosetti et al. (2013). The experiments have been conducted in a water channel for low cylinder aspect ratio without using endplates to vary the aspect ratios of 13 to 0.5 (Figure 1, see Rahman et al. 2013 for details experimental approach). The study was designed based on low aspect ratio structure such as circular FPSO, Spars, and many other circular floating structures. Of particular interest were to measured VIV amplitude responses, hydrodynamic forces, frequency response, and Strouhal number. Some of the results from the experiments were included and briefly explained here. The results shows that VIV was freely developed on the high aspect ratio structure and completely disturbed and eliminated for aspect ratio of 0.5 (Figure 2). Numerical simulation of these cases would provide an additional explanation in the context of pressure and vortex formation for the reduction of the responses as the aspect ratio reduced. The Strouhal number have been plotted with results from Gouda (1975) in Figure 3 and shows continuation of lower values of Strouhal number as the aspect ratio reduced down to 0.5 . The reduction was due to three dimensional wake 
which triggered turbulent region and resulting in reduction of vibration amplitude, hence reduced the frequency of vortex shedding.

In this paper, continuous effort has been undertaken to study the fluid-structure interaction of low aspect ratio offshore structure (i.e., circular FPSO and spars) using numerical simulation in order to complement previously published paper by Rahman et al. (2013). The significance of this study is to strengthen and compare the numerical results with the experimental investigation of a range of low aspect ratio in water without the use of end plates. It should be noted that there were some limitations in the experiments conducted by Rahman et al. (2013). For example, there are some limitations on the flow visualization to capture the effect of the aspect ratio on the occurrence of VIV. Additionally, the measurement of the parameters during experiments solely depended on the measurement devices. Hence, the wake pattern, pressure distribution, forces distribution and correlation length of the VIV cannot be fully visualized and captured. Clarifications on these important characteristics were needed in order to support the result collected from the experiments. Three-dimensional effects are also expected to show an important role in varying the Strouhal frequency. Three experimental cases were selected for the numerical simulation which are $L / D=$ 13, 5, and 1. 


\section{Model description}

\subsection{Governing equations}

The simulations considered an unsteady, incompressible fluid flow past a cylinder in a fluid channel. The CFD models were developed as an approximation method in order to compare with the experimental data. Fluid motion is governed by continuity and Navier-Stokes equations, given by

$$
\text { Continuity } \quad \nabla \cdot u=0
$$

$$
\text { Momentum } \quad \rho\left(\frac{\partial u}{\partial t}+u \cdot \nabla u\right)=-\nabla p+\mu \nabla^{2} u+f
$$

where $\rho$ is the fluid density, $u$ is the flow velocity vector, $p$ is the pressure and $f$ represents the force acting on the fluid.

\subsection{Turbulence model}

The approach used in this study was a Reynolds Average Navier Stokes (RANS). By using RANS, the velocity is decomposed and the Navier-Stokes terms. Equations (1) and (2) are rewritten into a time averaged form.

$$
\begin{gathered}
\frac{\partial U_{i}}{\partial x_{i}}=0 \\
\frac{\partial U_{i}}{\partial t}+U_{j} \frac{\partial U_{i}}{\partial x_{j}}=-\frac{1}{\rho} \frac{\partial P}{\partial x_{i}}+\frac{\partial}{\partial x_{i}}\left[\left(v+v_{t}\right)\left(\frac{\partial U_{i}}{\partial x_{j}}+\frac{\partial U_{j}}{\partial x_{i}}\right)\right]+g_{i}
\end{gathered}
$$

where $U$ is the velocity averaged over time $t, \rho$ is the fluid density, $P$ is the pressure, $v$ is the kinematic viscosity, $v_{t}$ is the eddy viscosity and $g$ is the gravity acceleration. Turbulence model of $k$ - $\omega$ was used for the simulation after some validation processes. The $k$ - $\omega$ turbulence model was selected based on the types of flow and accuracy level on the boundary velocity profile and wake. 


\subsection{Boundary Conditions}

The boundary conditions defined on the CFD model domain were:

(1) A uniform mean velocity at the inlet plane, $U=U_{m}$, where $U_{m}$ is the mean velocity of the uniform flow.

(2) A prescribed mesh displacement in $y$-axis, $y=A \sin 2 \pi f_{v} t$ specified on the cylinder boundary wall. (refer section 2.3.1)

(3) Slip wall assigned to the wall boundaries to simulate the towing conditions from the experiments, where no shear forces occurred between the fluid and the walls $\left(U=U_{m}\right)$

The outlet plane for the geometry was set as pressure outlet boundary, where no viscous stress was considered $\left(P_{o}=0\right)$. Figure 4 shows the CFD model domain and boundary conditions used in present simulations. The width of the domain was defined as $1.25 \mathrm{~m}$ and the height was $1.1 \mathrm{~m}$. The actual length of the towing tank used in our experiment was $50 \mathrm{~m}$. However, in order to simplify the model geometry and optimize the number of elements, the channel length was limited to $2.5 \mathrm{~m}$. The length of the domain, which was around $30 D$ in the wake region (behind the cylinder), proved to be sufficient in capturing the vortex-shedding phenomena after a series of simulations. The cylinder was centred at $0.5 \mathrm{~m}$ from the inlet and $0.625 \mathrm{~m}$ from the side walls in order to ensure the fluid flow uniformly past the cylinder with no nonphysical solution acquired.

During the simulation, the cylinder aspect ratio was varied according to each selected case. The submerged length was defined as $L$, where the distance was measured from the water surface to the end of the cylinder. No free surface effects were included in the CFD simulation. The boundary of the free surface was defined as a slip wall $(U=$ $\left.U_{m}\right)$. This is due to the complexity of the free surface behaviour such as wave run-up and wake disturbance that might require a high computational cost and as an alternate 
modelling approaches. However, the simulations were focussed more on the hydrodynamic responses, correlation length of the shedding and end conditions' effects. The effects of these assumptions will be further discussed in this paper.

The aspect ratios used in the simulation were $L / D=13, L / D=5$, and $L / D=1$ based on the experiments by Rahman et al. (2013) on the aspect ratio effects, varying $L / D=0.5$ to 13 . The selection of the corresponded cases was based on the variation of high to low aspect ratio. Table 1 shows the cylinder size and the natural frequency in the still water for the case studied.

\subsubsection{Oscillating cylinder boundary conditions}

A forced oscillation simulation has been developed for this CFD model. The cylinder is assumed to exhibit sinusoidal oscillations with constant amplitude and frequency. Mesh displacement has been prescribed on the cylinder boundaries as a sinusoidal oscillation to imitate the vibration behaviour observed in the experiments. The displacement was expressed as,

$$
y=A \sin 2 \pi f_{v} t
$$

where $y$ is the displacement of the cylinder, $A$ is the amplitude of vibration and $f_{v}$ is the vibration frequency. The amplitude and frequency of the vibration were taken from the experimental measurements for a range of reduced velocities. The drag and lift coefficients were calculated from the forces acting on the cylinder boundaries. The cylinder was forced to vibrate at specific amplitude and frequency in a specific flow velocity in order to calculate the hydrodynamic forces as illustrated in Figure 4. A moving mesh boundary was used to vibrate the cylinder in the transverse direction of the flow. In doing so, it became evident that some of the mesh elements might become inverted and truncated due large deformation. The accuracy of the solution depreciates and may lead to divergence of the solver. Winslow smoothing was used to encounter the 
deformed mesh instability. This smoothing method was selected due to its stability compared to other smoothing method. However, the downside was that the smoothing slowed down the computations and was memory consuming. The smoothing was done by solving equations for the mesh displacements

$$
\frac{\partial^{2} X}{\partial x^{2}}+\frac{\partial^{2} X}{\partial y^{2}}=0, \quad \frac{\partial^{2} Y}{\partial y^{2}}+\frac{\partial^{2} Y}{\partial x^{2}}=0
$$

where $x$ and $y$ are the spatial coordinates of the spatial frame, and $X$ and $Y$ are the reference coordinates of the material frame (Pryor 2010).

\subsection{Computational meshing}

In the present simulations, a structured grid mesh consisting of hexahedral and mapped mesh has been used (Figure 5). The area around the cylinder and the wake region was refined using a quadrilateral mesh to capture the reaction forces around the cylinder. It is known that the separation of the vortices initiates on the wall of the cylinder, hence refinements may be needed in the area to capture the vortex-shedding phenomenon during lock-in. The complete meshes for the geometry consisted of 856,000 mesh elements. The final mesh quality was based on a series of convergence test to determine optimum mesh elements. For a given cell count, a hexahedral mesh will give accurate solutions, especially when the grid lines are aligned with the flow. In all CFD simulations, the mesh density should be high enough to capture all relevant flow features. Poor mesh quality may also affect the computational time and the convergence of the simulation. Skewness, smoothness in the changing of the cell size, and the cell aspect ratio are the important criteria to ensure the model has a high quality mesh. In the present model, the cell aspect ratio (width to length ratio) was kept close to one $(b / l \approx 1)$ as illustrated in Figure 5(b). The symmetrical shape of grid elements was found to improve the convergence rate during the simulation. 
Mesh transition between domains also needs to be considered. Smooth cell size transitions created a smooth transition of the flow during the simulation. The number of elements in the model was controlled using the present meshing method. The region near the cylinder walls was refined to capture the flow physics effectively. Note that the boundaries near the body were dense compared to other domain regions. Additionally, the higher density throughout the cylinder depth (z-axis) was expected to produce highquality results.

\section{Model validation}

The validation process was undertaken in order to ensure the validity and reliability of the present CFD model. The model was validated against published literature of Schafer and Turek (1996) on 2-Dimensional (2D) and 3-Dimensional (3D) flow past a cylinder. Dimensions and the definition of boundary conditions for the present 2D and 3D models are shown in Figure 6. The flow velocity in the domain channel was uniform, and incorporated with the mean velocity, $U_{m}$. The simulations were conducted for a 7second period with a time step of 0.001 . Table 2 shows the results for the different cases simulated for the validation purposes. Based on the results acquired, there was a good agreement on the drag and lift coefficient values between our models and the published values from literature and were in the proposed range. In the present model, a fully coupled and direct solver was used. The simulation was converged around 200 iterations or 150 time-step. Figure 7 shows the drag and lift coefficient time-history plots for 2D-1 and 2D-2 model respectively, plotted with the values from literature. The pressure difference between two points on the cylinder was also computed from the simulation. The two points were defined as the stagnation point, point $1(0.15,0.2)$, and the end point of the cylinder, point $2(0.25,0.2)$. Figure 8 shows the pressure difference 
in case 2D-1 and 2D-2 was in excellent agreement with the solution by Schafer and Turek (1996).

\section{Results \& discussions}

\subsection{Stationary cylinder simulation}

The simulations were conducted using a stationary cylinder before proceeding to the forced vibration cylinder. The results of the 3D fixed cylinder case are presented in Figure 9 and compared with the experimental data of Wieselbeger (1921) and Schewe (1983). The simulation for the stationary cylinder was conducted using similar domain, mesh density and non-dimensional time step used for forced vibration simulations. The Reynolds number was in the subcritical flow region $\left(1.1 \times 10^{4}<R e<2.3 \times 10^{5}\right)$. The drag values for $L / D=13$ showed a good agreement with the literature, which varied from 1.18 to 1.27 . It can be seen that the mean drag coefficients for the $L / D=5$ model obtained from the simulations showed lower values compared to $L / D=13$ and the literature. The mean drag varied from 0.9 to 1.046 for $L / D=5$ model and 0.89 to 0.95 for $L / D=1$ model. The decrease of the mean drag values was expected due to the decrease of the aspect ratio as discussed in Rahman et al. (2013) in the context of the experimental results.

\subsection{Forced Oscillating Cylinder Simulations}

3D CFD models were created for three different aspect ratios proposed before, namely $L / D=13,5$, and 1, matching the experimental conditions of Rahman et al. (2013). Ten velocity increments were selected from the experimental data used in each simulation. Table 3 shows the experimental data for velocity, amplitude and vibration frequency. These parameters were used to fit in the equation (5) for the forced vibration simulation. 


\subsubsection{Amplitude time history}

The simulations were configured using the parameters in Table 3. The cylinder was forced to vibrate at a certain amplitude and frequency while under different flow velocities. Examples of the amplitude time history of the CFD simulations during the lock-in for all aspect ratios investigated are plotted in Figure 10 at reduced velocities of $5.45,8.2$, and 6.6 respectively. The time series were compared to the time series from the experiments. It can be seen that the plots were in a good agreement with each other. However, due to the beating motion in the time series obtained in the experiments, it is expected to see slightly lower values of amplitude over the time in the plot. It should be noted that the frequency of the vibration from the experiment 1 and 4 almost matched up with the CFD plot while a slightly delayed motion was observed in experiment 7 $(L / D=1)$.

\subsubsection{Drag coefficients}

The time histories of the drag coefficients for the CFD model are compared with the experimental time histories in Figure 11. As seen from the plot, there is an unsteady oscillation captured by the strain gages from the experiment. It was probably due to restrained movement of the cylinder in inline direction. The experimental setup was designed mainly to capture transverse movement and the data from the strain gages in the inline direction were possibly disturbed by the vibration in the transverse direction due to VIV. Figure 12 presents the plots for the mean drag coefficients from forced oscillation simulations compared with experiments. In experiment $1(L / D=13)$, it can be seen that the CFD simulation showed a similar pattern with the experimental data. The peak value from the CFD model almost matched up with the experimental value which occurring at 1.95 and 1.99 respectively, but showed a slight discrepancy at higher reduced velocity $U_{\mathrm{r}}$. Higher drag values were captured from the CFD at 0.8 compared to 
the measurement from the experiments 0.501 at the earlier $U_{r}$. A slightly lower value of $C_{\text {Dmean }}=0.85$ from the CFD captured during the lock-out compared to $C_{\text {Dmean }}=1.25$ from the experiment. However, there were similarities in the curved shape and pattern compared with the experiment. The differences were expected due to a different oscillation captured as mentioned earlier.

In experiment $4(L / D=5)$, the results of the simulation showed a lower value of drag at 0.757 compared to experimental value, 0.9153 except at $U_{r}=6.92$ where the $C_{\text {Dmean }}$ was observed at 1.55 , slightly higher than the experimental value of 1.4617 . The CFD simulation captured the curved shape of the $C_{\text {Dmean }}$ plot, however underestimated the value of the drag coefficients over the reduced velocity. The mean drag plot from the experiment peaked at 1.68 compared to 1.6 measured in the CFD model. A poor agreement can be seen from the plot for experiment $7(L / D=1)$ where $C_{\text {Dmean }}$ from the CFD model was significantly higher compared to the experimental data. The peak of the mean drag coefficient was measured at 0.95 for the simulation compared to 0.42 from the experiment. This is probably due to three-dimensional effects captured at the lower end of the cylinder during the simulations. Additionally, the free surface effects were not captured due to limitations on the boundary conditions mentioned earlier, which could contribute to the differences.

\subsubsection{Lift coefficients}

Examples of the root mean square lift coefficients time history are plotted in Figure 13 and compared with the time history from experiments. The CFD model captured a slightly higher value of lift coefficients during the simulations compared with the experiments time history. This higher value was expected due to the higher amplitude captured, as explained previously. The $C_{\text {Lrms }}$ was measured from the CFD model by integrating the boundaries of the cylinder. The $C_{\text {Lrms }}$ plot is illustrated in Figure 14. In 
experiment $1(L / D=13)$, the CFD value from the simulation peaked at 3.01 compared to the experimental value at 2.704 . The simulation data followed the curve of the $C_{\text {Lrms }}$ with slightly higher values over the reduced velocities. A good agreement was observed for $L / D=13$ and 5. However, the influence of the 3D effects from free surface and end condition was observed for the low aspect ratio of $L / D=1$ where the plot shows a slightly lower value compared to the experiment.

The results of the CFD simulation and the experiment $4(L / D=5)$ are plotted together in the Figure 14. A higher response was captured in the CFD model compared to the responses measured in the experiment. The $C_{L r m s}$ was steadily high at lower velocity and slightly reduced after reaching the maximum value of 1.87 . The values at the higher reduced velocities were still quite high compared to the experimental data. As for the lowest aspect ratio in the simulation $(L / D=1)$, the CFD simulations seemed to capture a higher lift force response compared to the experimental data. However, the

simulation data followed the shape of the curve plotted from experiment 7 . The frequency spectra for the simulations were illustrated in Figure 15 with the vibration frequency of $0.98 \mathrm{~Hz}, 0.98 \mathrm{~Hz}$ and $0.51 \mathrm{~Hz}$ respectively.

\subsubsection{Flow behaviour}

An example of velocity magnitude and pressure contour are plotted in Figure 16. A different velocity region during the VIV phenomenon was observed during the simulations. The velocity was reduced in the wake region behind the cylinder compared to a higher velocity around the cylinder as expected. The velocity magnitude in the wake region shows a decrease in velocity as the vortex shedding moved downstream. The energy from the vortex shedding diminished over the simulation time as the vortices shed away from the cylinder. 
The 3D effects were observed during the simulations. Cylinders of varying submerged depth allow different wake disturbance in the fluid structure interactions. Simulation of $L / D=1$ shows a smaller interaction area which simulates the experimental condition. End conditions were dominant for lower aspect ratio cylinder due the distance between the bottom-end of the cylinder with the free surface. Fairly good agreements were captured previously for higher aspect ratios on the hydrodynamics forces.

Meanwhile, for a lower aspect ratio, a poor agreement was observed. This was due to the $3 \mathrm{D}$ effects of the VIV at the bottom end of the cylinder. Formation of the vortex shedding disturbed by the cylinder end which lead to the reduction of the structure vibration.

It can be seen from the experiment $7(L / D=1)$ simulation, the cylinder length is relatively small compared to the height of the domain. The flow outside the wake region and below the cylinder remained uniform throughout the simulation. However, the vortex-shedding was still noticeable for this aspect ratio, with lower magnitude of vibration observed. The pressure gradient influenced the formation of the vortex shedding. A uniform vortex shedding could be formed along the cylinder length, where the low pressure of the wake region generated, as the flow past the cylinder. However, a low aspect ratio cylinder $(L / D=1)$ shows a relatively small low-pressure area at the downstream of the cylinder which supported by the discrepancy in the data compared to the experiment. It can be seen that the intensity of the vortices diminished with decreasing aspect ratio. The formation of the vortex shedding depends on the correlation length of the cylinder. For a high aspect ratio cylinder, vortex shedding generated uniformly along the cylinder with minimal disturbance at the lower end of the cylinder. Meanwhile, for low aspect ratio cylinder, a significant disturbance from the cylinder 
bottom end were observed both in experiment and simulation. The end effects affected the intensity of the vortices. Note that from the plots, the vortices shed from the edge of the cylinder and diminished as they travel downstream. The vortices during the VIV for lower aspect ratio cylinder did not sustain long after shedding from the cylinder. This was also probably because of the lower energy to contain the vortices before being washed out.

Normally, the vortex cells separation were uniformly generated along the span during the VIV for an infinite long structure (Constantinides and Oakley 2006). However, one can observe the reduction of the vortex cells' length as the vortices separated away from the low aspect ratio cylinder. The isosurfaces of velocity were plotted for various aspect ratios in Figure 17. The vortex cells' length reduced significantly to almost half of the cylinder length over time. It can be concluded from this simulation that the correlation length of the vortex cell was disturbed by the end condition of the cylinder. If the cylinder was positioned close to the bottom of the channel, a minimal or no disturbance would be observed. The bottom of the channel will act as a big end plate to the cylinder. However, the cylinder was positioned far from the bottom of the channel to minimize blockage ratio and to capture the end effects on the VIV.

As mentioned before, the boundary condition for the free surface was defined as the slip wall $\left(U=U_{m}\right)$ or a 'clean' free surface (Morse 2005). The effects of the free surface on the VIV were assumed negligible due to the limitation of computational costs. The surface defined as a uniform flow boundary with no disturbance allowed. However, the free surface was observed in the experiments significantly affect the formation of the vortex cells along the cylinder. This will affect the force acting on the cylinder, hence reduced the total force. Disturbance of the vortex shedding formation was significant if 
the cylinder close to the free surface. Hence, for a low aspect ratio cylinder, most of the length of the cylinder was close to the surface which lead to a high disturbance along the cylinder compared to a high aspect ratio cylinder (Rahman et al. 2010). A combination of end effect and free surface effects were significant to a low aspect ratio. This supported previous data on drag and lift forces where a significantly higher value captured from CFD compared to the experiments. The inclusion of the free surface effects will be considered in the future in order to comprehensively validate the experiments for low aspect ratio cylinder.

Earlier results on the effects of the aspect ratio from the experiments found a reduction of the amplitude of vibration as the aspect ratio reduced (Rahman et al. 2013). This is at least partially due to the end condition effects observed in this simulation. This result could possibly make a strong relationship with the reduction of the vibration amplitude in the experiment discussed earlier. The flow around the end of the cylinder was in high velocity compared to the wake region. The difference in the velocity profile was observed where a moderate velocity flow layer was developed between the vortex cell and the flow from the cylinder end. The layer was observed to create a disturbance on the vortex cell development over the duration of the VIV phenomenon. A similar phenomenon was observed in $L / D=5$ cylinder simulation where the development of the vortex cell was disturbed by the end of the cylinder. The vortex cell narrowed in the downstream direction. The energy from the vortex shedding was also weakened due to the flow disturbance from the end condition. The reduced correlation length is also one of the main reasons of the lower energy produced from the vortex shedding. The length of the cylinder is small; hence, a small vibration was generated. The end conditions tend to dominate for th (c) r aspect ratio, with major disturbance in the wake development as shown in Figure 17. 
Based on our assumptions earlier, one should expect a significant reduction in the vibration amplitude if the free surface effect was considered. Disturbance at the free surface in the wake region, pressure variation at the front and aft of the cylinder and the occurrence of water run-up are several factors that influenced the formation of the vortex cells. It can be seen from the simulation results that the vortex cells were significantly reduced after the vortices shed from the cylinder. The vortex cells were disturbed by the 3D flow and hence reduced in size as the vortex cell moved downstream. The velocity magnitude represented in the plot as a comparison of the vortex formation along the cylinder length. It can be seen from Figure 18, the magnitude of vortices reduces as the aspect ratio reduces. End conditions affected the vortex formation where the correlation length reduced as the vortex shedding move downstream. The conclusion can be made that the aspect ratio, the free surface and the end conditions of the cylinder are the important factors of the variation in the vibration amplitude based on the observations made in the CFD simulations.

The effect of aspect ratio on the VIV phenomenon is apparent in visualizations of pressure distributions, correlation length, wake formation and vortex shedding pattern captured from the forced vibration CFD simulations. The correlation length was observed to be higher for higher aspect ratio $(L / D=13)$ and proportionally decreased as the aspect ratio decreased. The presence of the turbulence in the flow reduces the correlation length as investigated by Novak and Tanaka (1977). Turbulence in the flow is known to be one of the significant factors in the event of VIV. The correlation length also changes with Reynolds number for a smooth cylinder (Sumer and Fredsoe 2010). The vortex cells draw energy from the vortices, producing higher forces to vibrate the structure. 3D effects at the end of the cylinder influenced the development of the vortex cells as the $L / D$ reduced. It is known that the free surface is important when designing 
any floating structure near the still water level (SWL). In real sea conditions, the wavefree surface of the structure continually changes the submerged part of the structure about the SWL (Chakrabarti 2005). However, in the present work, the wave and free surface effects have been captured in the experiments but not truly captured in the simulations. This arrangement will definitely affect the combination of the forces on the structure in comparison with the experiments and phenomenological model.

\section{Conclusion}

The combined observations of the experiments and CFD simulations has proven the importance of studying the effect of the aspect ratio for the floating structure. High aspect ratio $(L / D=13$ to 5$)$ can represent offshore spars platform and the lower aspect ratio $(L / D=3$ to 0.5$)$ represent any short structure such as circular FPSO. The effects of end condition, the free surface, and the flow profile was clearly observed when comparing the results from the experiments and the CFD simulations. The results of the experiments and the simulations have shown that the structure with higher aspect ratio encounter higher vibration amplitude compared to lower one. The behaviour of the vortex shedding depends on the aspect ratio examined. This is supported by the experimental data published by Rahman et al. (2013). The VIV response proportionally reduces as the aspect ratio reduces. It can be concluded from the simulations that the intensity of the vortices for $L / D=13$ was higher compared to $L / D=5$ and 1 . The vortex intensity represents the VIV response on the particular cylinder when subjected to the flow. $L / D=1$ shows a significant lower vortex intensity, and the vortices did not manage to establish a uniform periodic pattern. The vortices shed from the edge of the cylinder and diminished instantaneously. In terms of practical application, the circular FPSO is more stable and known to be exhibited higher stability compared to any conventional FPSO (Major and Eggan 2009). However, many other factors need to be 
considered to conduct a thorough analysis on the structure such as the size, shapes, wave and current profile, buoyancy, mooring types, and also VIV mitigations.

Further study involves on numerical simulations of free vibration cylinder with inclusion of free surface, end condition, and aspect ratio effects on VIV. Predictions of the free vibration CFD simulation will be discussed and compared with the experimental data in order to establish a relationship describing VIV as a factor of aspect ratios.

\section{References}

Bearman PW. 1984. Vortex shedding from oscillating bluff bodies. Annual review of fluid mechanics. 16:195-222.

Blevins RD. 1990. Flow-induced vibration. New York: Van Nostrand Reinhold.

Carberry J, Govardhan R, Rockwell D, Williamson CHK. 2004. Wake states and response branches of forced and freely oscillating cylinder. European J Mechanics B/Fluids. 23:89-97.

Chen SS. 1987. Flow induced vibration of circular cylindrical structures. Springer, Washington DC, US: Hemisphere Publishing Corporation.

Chakrabarti SK. 2005. Handbook of offshore engineering. Amsterdam; London: Elsevier, ISBN 978-0-08-052381-1.

Gonçalves RT, Franzini GR, Fujarra ALC, Meneghini JR. 2010. Two degrees-of-freedom vortex-induced vibration of a circular cylinder with low aspect ratio. In Sixth Conference on Bluff Body Wakes and Vortex-Induced Vibrations-BBVIV. Capri Island, Italy.

Gonçalves RT, Fujarra ALC. 2014. Experimental Study on Vortex-Induced Vibration of Floating Circular Cylinders with Low Aspect Ratio. Proceedings of the ASME $33^{\text {rd }}$ International Conference on Ocean, Offshore and Arctic Engineering.

Gouda BHL. 1975. Some measurements of the phenomena of vortex shedding and induced vibrations of circular cylinders. Technische Universitat Berlin Report. 7501. 
Halkyard J, Atluri S, Sirnivas S. 2006. Truss spar Vortex Induced Motions: Benchmarking of CFD and model tests. Proceedings of the ASME $25^{\text {th }}$ International Conference on Offshore Mechanics and Arctic Engineering. Hamburg, Germany.

Holmes S, Constantinides Y, Owen HO Jr. 2006. Simulation of riser VIV using fully three dimensional CFD simulations. Proceedings of the ASME $25^{\text {th }}$ International Conference on Offshore Mechanics and Arctic Engineering. Hamburg, Germany.

Holmes S. 2008. Predicting spar VIM using CFD. Proceedings of the ASME of $27^{\text {th }}$ International Conference on Offshore Mechanics and Arctic Engineering. Estoril, Portugal.

Kamarudin MH, Thiagarajan KP. 2010. Investigation of fluid-structure interaction on controlled oscillations of a cylinder at moderate Reynolds number. Proceedings of the ASME $29^{\text {th }}$ International Conference on Offshore Mechanics and Arctic Engineering. Paper 2010-20918. Shanghai, China.

Major F, Eggan S. 2009. Significant benefits with a cylindrical hull for FPSO, drilling, LNG, and offshore power plant applications. Petrotech. Delhi, India.

Morse TL, Govardhan RN, Williamson CHK. 2008. The effects of end conditions on the vortex-induced vibration of cylinders. J Fluids Struct. 24:1227-1239.

Novak M, Tanaka H. 1977. Pressure correlations on a vibrating cylinder. Proc. $4^{\text {th }}$ Int. Conf. on Wind Effects on Building and Structures. Heathrow, U.K., Edby K.J. Eaton. Cambridge Univ. 227-232.

Oakley Jr OH, Navaro C, Constantinides Y, Holmes S. 2005. Modeling Vortex Induced Motions of spars in uniform and stratified flows. Proceedings of the ASME $24^{\text {th }}$ International Conference on Offshore Mechanics and Arctic Engineering. Halkidiki, Greece.

Pantazopolous MS. 1994. Vortex-induced vibration parameters: critical review. Proceedings of the ASME $13^{\text {th }}$ International Conference on Offshore Mechanics and Arctic Engineering, 1:199-255.

Pryor RW. 2009. Multiphysics Modeling Using COMSOL: A First Principles Approach. Jones and Barlett Publishers, LLC. 
Rahman MAA, Thiagarajan KP. 2013. Vortex-Induced Vibration of Cylindrical Structure with Different Aspect Ratio. Proceedings of the ISOPE $23^{\text {rd }}$ International Offshore and Polar Engineers. Alaska, USA.

Rahman MAA, Esakki M, Kamarudin MH, Thiagarajan KP. 2010. Free Surface Effects on Vortex Induced Vibrations of Cylindrical Offshore Structures. Proceedings of the $6^{\text {th }}$ Australasian Congress on Applied Mechanics. Perth, W.A. 936-943.

Rosetti G, Vaz G, Hoekstra M, Goncalves RT, Fujarra ALC. 2013. CFD Calculations for Free-Surface-Piercing Low Aspect Ratio Circular Cylinder with Solution Verification and Comparison With Experiments. $32^{\text {nd }}$ International Conference on Ocean, Offshore and Arctic Engineering. Nantes, France.

Roshko A. 1961. Experiments on the flow past a circular cylinder at very high Reynolds number. J Fluid Mechanics. 10 (3): 345-356.

Sarpkaya T. 2004. A critical review of the intrinsic nature of vortex-induced vibrations. J Fluids Struct. 19(4): 389-447.

Schafer M, Turek S. 1996. Benchmark computations of laminar flow around a cylinder. NNFM 52 Flow Simulation on High Performance Computers II. Vieweg, Braunschweig. 547-566.

Sumer BM, Fredsoe J. 1997. Hydrodynamics around cylindrical structures. Singapore: World Scientific.

Thiagarajan KP, Constantinides Y, Finn L. 2005. CFD Analysis of Vortex Induced Motions of Bare and Straked Cylinders in Current. Proceedings of the ASME $24^{\text {th }}$ International Conference on Offshore Mechanics and Arctic Engineering. Halkidiki, Greece.

Wieselsberger C. 1921. Neuere Feststellungen iiber die Gesetze des Fliissigkeits- und Luftwiderstands. Phys. Zeitschr. 22: 321-8.

Wilcox DC.1998. Turbulence Modeling for CFD. 2nd ed. DCW Industries.

Willden RHJ, Graham JMR. 2001. Numerical prediction of VIV on long flexible circular cylinders.J Fluids Struct. 15: 659-669.

Williamson CHK, Govardhan R. 2008. A brief review of recent results in vortex-induced vibrations. J Wind Eng Ind Aerodynamics. 96: 713-735. 


\section{List of Tables}

Table 1 CFD model test cases. (Adapted from Rahman et. al 2013)

Table 2 Results of validation simulations.

Table 3 Experimental values for CFD $L / D$ simulations. (Extracted from Rahman et al. 2013) 
Table 1 CFD model test cases. (Adapted from Rahman et al. 2013)

\begin{tabular}{lccc}
\hline Aspect ratio, $L / D$ & Length, $L(\mathrm{~m})$ & Diameter, $D(\mathrm{~m})$ & Natural frequency, $f_{n}(\mathrm{~Hz})$ \\
\hline 13 & 0.78 & 0.06 & 0.99 \\
5 & 0.4 & 0.08 & 1.035 \\
1 & 0.16 & 0.16 & 0.543 \\
\hline
\end{tabular}


Table 2 Results of validation simulations.

\begin{tabular}{|c|c|c|c|c|c|c|c|c|}
\hline \multirow{2}{*}{\multicolumn{2}{|c|}{$\begin{array}{lc} & \\
& \\
\text { Case } & \mathrm{U}(\mathrm{m} / \mathrm{s}) \\
\text { No. } & (\mathrm{Re}) \\
\end{array}$}} & & \multicolumn{3}{|c|}{ Schafer \& Turek (1996) } & \multicolumn{3}{|c|}{ Present model } \\
\hline & & & $C_{D}$ & $C_{L}$ & $\Delta \mathrm{P}$ & $C_{D}$ & $C_{L}$ & $\Delta \mathrm{P}$ \\
\hline \multirow[t]{2}{*}{$2 \mathrm{D}-1$} & 0.3 & Max & 5.59 & 0.0110 & 0.1172 & 5.582 & 0.01093 & 0.11756 \\
\hline & $(20)$ & Min & 5.57 & 0.0104 & 0.1176 & & & \\
\hline \multirow[t]{2}{*}{$2 \mathrm{D}-2$} & 1.5 & Max & 3.24 & 1.01 & 2.46 & 3.2266 & 0.9953 & 2.42 \\
\hline & $(100)$ & Min & 3.22 & 0.99 & 2.50 & & & \\
\hline \multirow[t]{2}{*}{$3 \mathrm{D}-1 \mathrm{Z}$} & 0.45 & Max & 6.25 & 0.01 & 0.175 & 6.195 & 0.01 & 0.172 \\
\hline & (20) & Min & 6.05 & 0.008 & 0.165 & & & \\
\hline \multirow[t]{2}{*}{$3 \mathrm{D}-2 \mathrm{Z}$} & 2.25 & Max & 3.792 & 0.008 & - & 3.32 & -0.0086 & - \\
\hline & $(100)$ & Min & 3.225 & 0.01 & - & & & \\
\hline
\end{tabular}


Table 3 Experimental values for CFD $L / D$ simulations. (Extracted from Rahman et al. 2013)

\begin{tabular}{lccc|ccc|ccc}
\hline & \multicolumn{3}{c|}{ Exp. 1 $(L / D=13)$} & \multicolumn{3}{c|}{ Exp. 4 (L/D=5) } & \multicolumn{3}{c}{ Exp. $7(L / D=1)$} \\
No. & $U(\mathrm{~m} / \mathrm{s})$ & $A(\mathrm{~m})$ & $f_{v}(\mathrm{~Hz})$ & $U(\mathrm{~m} / \mathrm{s})$ & $A(\mathrm{~m})$ & $f_{v}(\mathrm{~Hz})$ & $U(\mathrm{~m} / \mathrm{s})$ & $A(\mathrm{~m})$ & $f_{v}(\mathrm{~Hz})$ \\
\hline 1 & 0.1215 & 0.0025 & 0.6823 & 0.1770 & 0.0008 & 0.8238 & 0.1791 & 0.0164 & 0.2058 \\
2 & 0.1791 & 0.0053 & 0.5859 & 0.2649 & 0.0012 & 0.7656 & 0.2398 & 0.0205 & 0.1916 \\
3 & 0.2398 & 0.0107 & 0.6333 & 0.3613 & 0.0015 & 0.6718 & 0.2974 & 0.0142 & 0.2553 \\
4 & 0.2974 & 0.0253 & 0.8395 & 0.4639 & 0.0452 & 0.7817 & 0.3613 & 0.0116 & 0.3443 \\
5 & 0.3236 & 0.0597 & 0.9766 & 0.5728 & 0.0698 & 0.9481 & 0.4199 & 0.0155 & 0.4568 \\
6 & 0.3613 & 0.0685 & 1.0729 & 0.6796 & 0.0757 & 0.9862 & 0.4650 & 0.0149 & 0.4773 \\
7 & 0.4639 & 0.0416 & 1.2156 & 0.7791 & 0.0538 & 1.1063 & 0.4985 & 0.0455 & 0.4915 \\
8 & 0.5383 & 0.0325 & 1.3113 & 0.8786 & 0.0274 & 1.2253 & 0.5728 & 0.0769 & 0.5133 \\
9 & 0.6189 & 0.0177 & 1.4424 & 1.0367 & 0.0147 & 1.1602 & 0.6451 & 0.0152 & 0.5599 \\
10 & 0.6796 & 0.0093 & 1.5726 & 1.1257 & 0.0102 & 1.0181 & 0.6796 & 0.0177 & 0.6006 \\
\hline
\end{tabular}




\section{List of Figures}

Figure 1 Experimental setup for the investigation of VIV on different aspect ratio (Rahman et al. 2013).

Figure 2 Normalized amplitude of different cylinder aspect ratio (Rahman et al 2013).

Figure 3 Strouhal Number against cylinder aspect ratio (Rahman et al 2013).

Figure 4 CFD model boundary conditions. The cylinder was forced to vibrate in transverse direction (to follow the Equation 5).

Figure $5 L / D=13$ geometry model with controlled hexahedral meshes (a) Plan view, (b) $3 \mathrm{D}$ view.

Figure 6 (Top) 2D and (Bottom) 3D domain with boundary conditions (Adapted from Shafer \& Turek 1996).

Figure 7 Hydrodynamic coefficients time history for 2D cases. Right: 2D-1 $(R e=20)$ and Left: 2D-2 $(R e=100)$.

Figure 8 Pressures at point 1 and 2 of the cylinder for 2D cases. Comparison of the pressure difference with the literature. Right: 2D-1 $(R e=20)$ and Left: $2 \mathrm{D}-2(R e=100)$. Figure 9 Mean drag coefficients for the stationary cylinder case. Comparison with the published results of Wieselberger (1921) and Schewe (1983).

Figure 10 Amplitude time series. Comparison between CFD simulations and experiments by Rahman et al. (2013). (a) Experiment $1(L / D=13)$ at $\mathrm{U}=0.3236 \mathrm{~m} / \mathrm{s}$ $\left(U_{r}=5.45\right)$. (b) Experiment $4(L / D=5)$ at $U=0.6796 \mathrm{~m} / \mathrm{s}\left(U_{r}=8.2\right)$. (c) Experiment 7 $(L / D=1)$ at $U=0.5728 \mathrm{~m} / \mathrm{s}\left(U_{r}=6.6\right)$. Beating phenomenon observed in the experimental data, captured in the forced vibration simulations.

Figure 11 Mean drag coefficients time series. Comparison between CFD simulations and experiments by Rahman et al. (2013). (a) Experiment 1 at $U=0.3236 \mathrm{~m} / \mathrm{s}\left(U_{r}\right.$ 
$=5.45)$. (b) Experiment 4 at $U=0.6796 \mathrm{~m} / \mathrm{s}\left(U_{r}=8.2\right)$. (c) Experiment 7 at $\mathrm{U}=0.5728$ $\mathrm{m} / \mathrm{s}\left(U_{r}=6.6\right)$.

Figure 12 Mean drag coefficients. Comparison between the aspect ratios results from CFD simulation and experiments by Rahman et al. (2013).

Figure 13 RMS lift coefficients time series. Comparison between CFD simulations and experiments by Rahman et al. (2013). (a) Experiment 1 at $U=0.3236 \mathrm{~m} / \mathrm{s}\left(U_{r}=5.45\right)$. (b) Experiment 4 at $U=0.6796 \mathrm{~m} / \mathrm{s}\left(U_{r}=8.2\right)$. (c) Experiment 7 at $\mathrm{U}=0.5728 \mathrm{~m} / \mathrm{s}\left(U_{r}\right.$ $=6.6)$.

Figure 14 RMS lift coefficients. Comparison between the aspect ratios results from CFD simulations and experiments by Rahman et al. (2013).

Figure 15 Frequency spectra from CFD simulations (a) Experiment 1 at $U=0.3236 \mathrm{~m} / \mathrm{s}$ $(U r=5.45)$. (b) Experiment 4 at $U=0.6796 \mathrm{~m} / \mathrm{s}\left(U_{r}=8.2\right)$. (c) Experiment 7 at $U=$ $0.5728 \mathrm{~m} / \mathrm{s}\left(U_{r}=6.6\right)$.

Figure 16 Velocity magnitude at $x$-z-plane with the pressure contour distribution. (a) Experiment $1(L / D=13)$ at $U_{r}=5.45$, (b) Experiment $4(L / D=5)$ at $U_{r}=8.2$, (c) Experiment $7(L / D=1)$ at $U_{r}=6.6$.

Figure 17 Isosurface of vorticity plot. (a) Experiment $1(L / D=13)$ at $U_{r}=5.45$, (b) Experiment $4(L / D=5)$ at $U_{r}=8.2$, (c) Experiment $7(L / D=1)$ at $U_{r}=6.6$. (Isosurface level. Above: Level 5. Bottom: Level 20.

Figure 18 Vortex shedding formation and wake formation of three different aspect ratios. (a) $L / D=13$, (b) $L / D=5$, (c) $L / D=1$. 

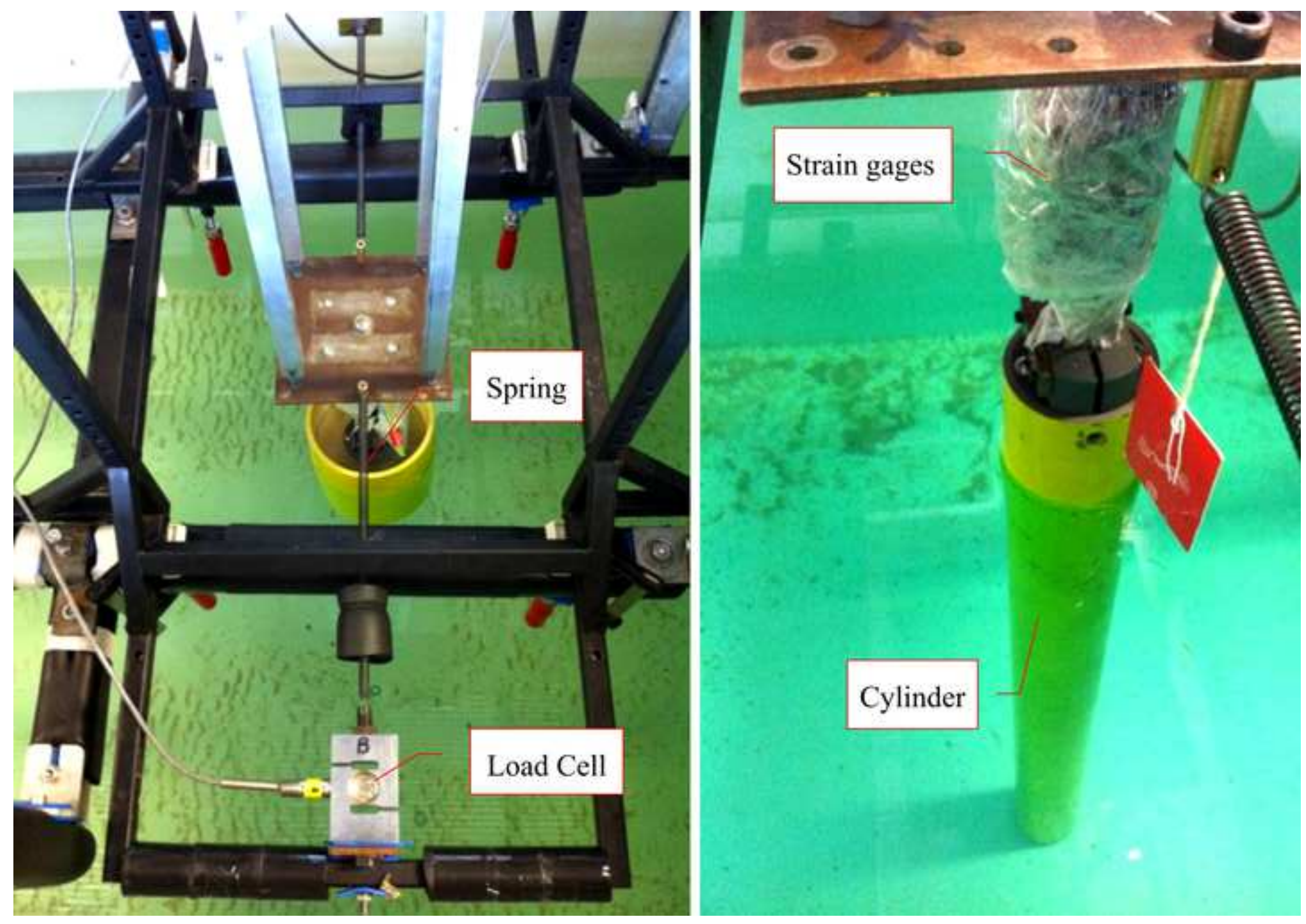


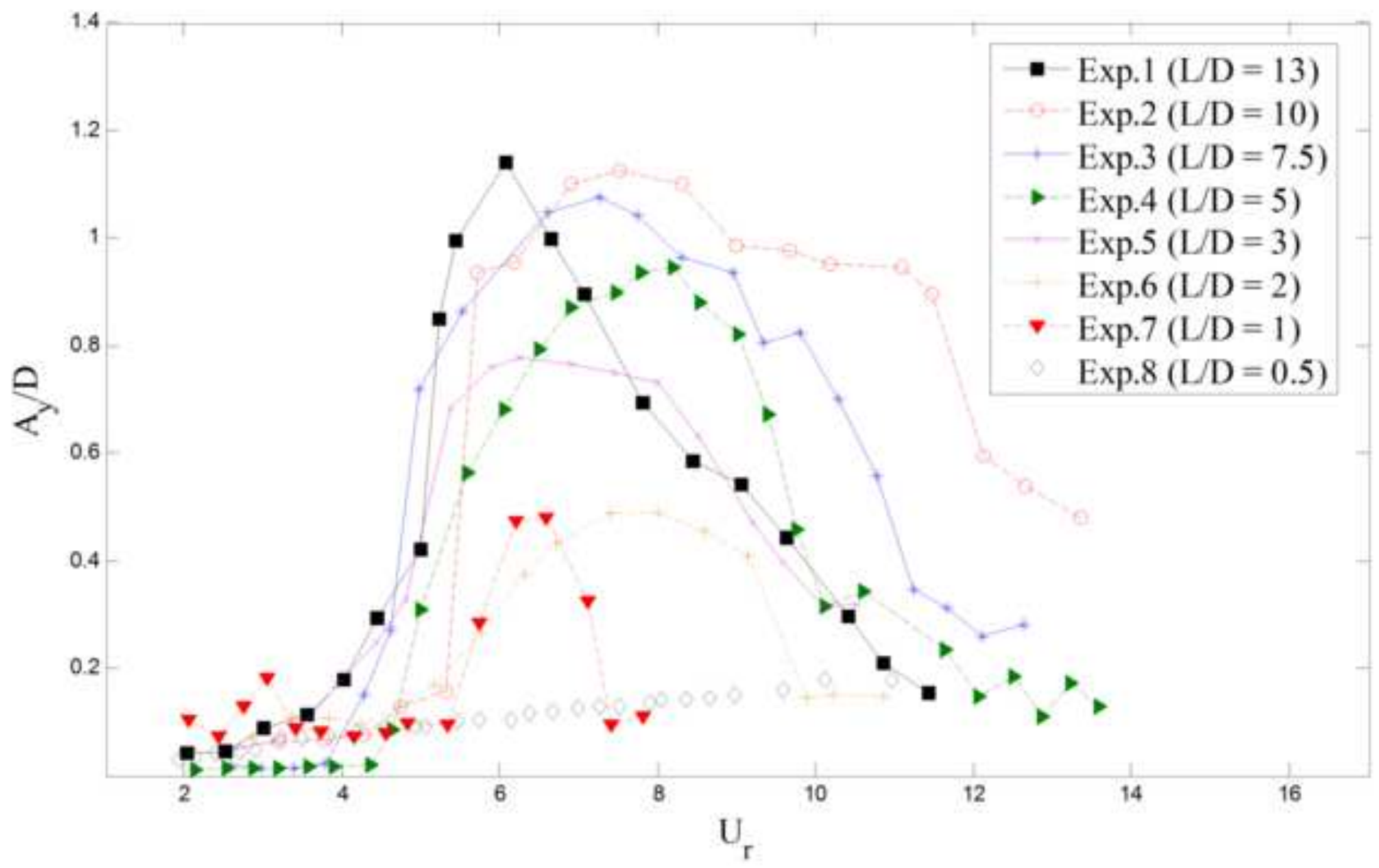




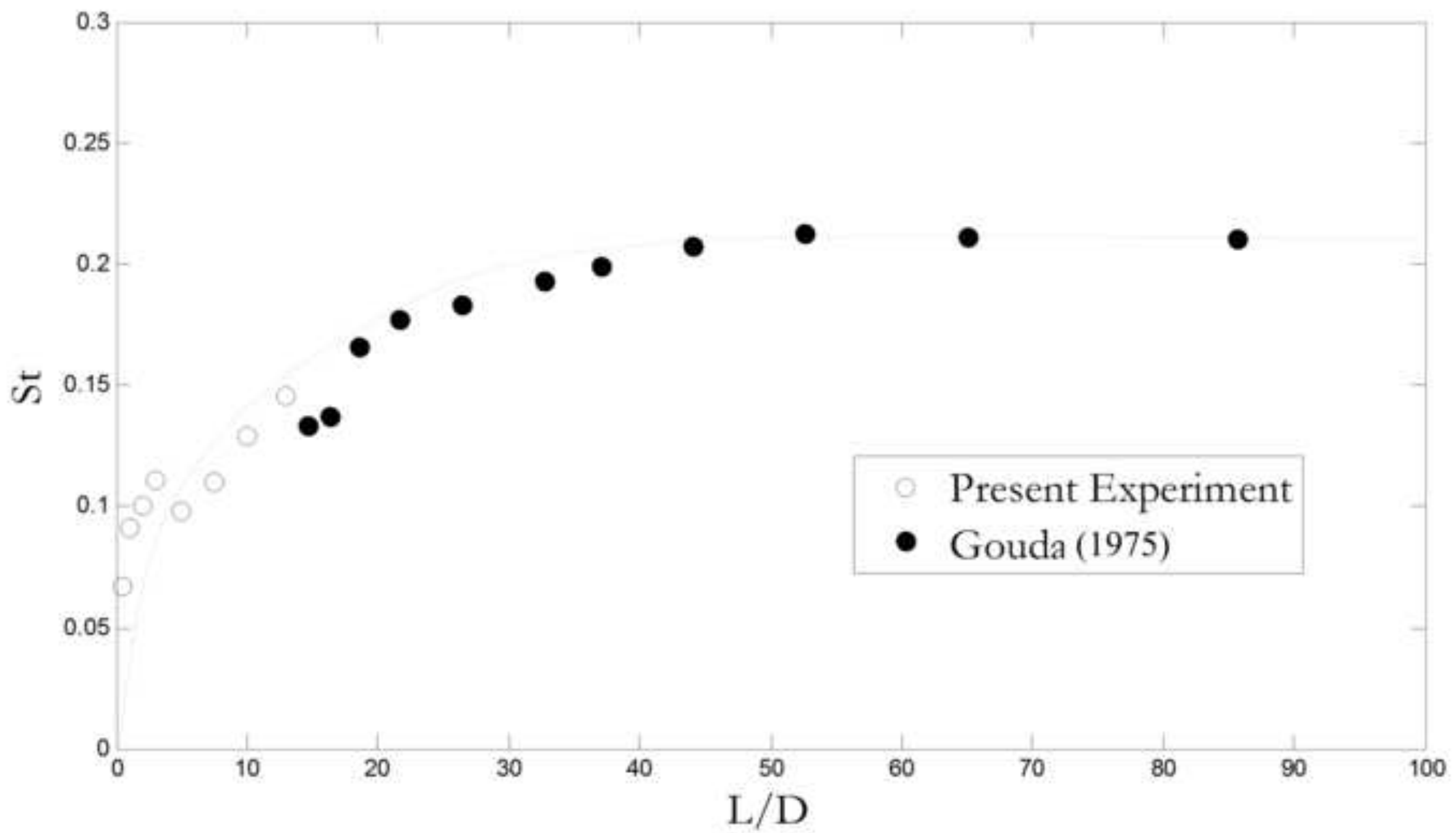


Figure 4
Click here to download high resolution image

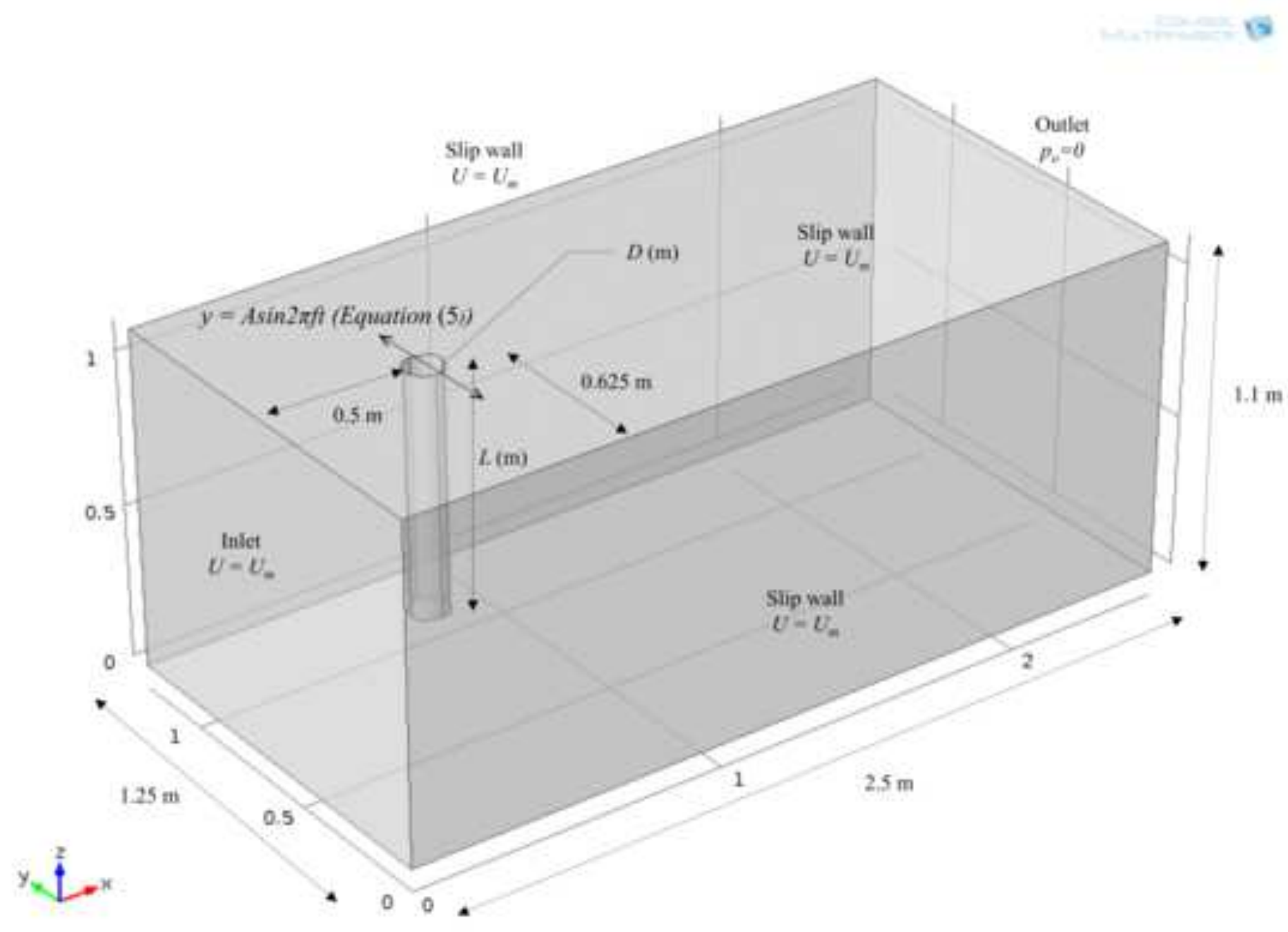
m.

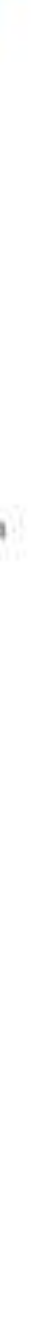


Figure 5
Click here to download high resolution image
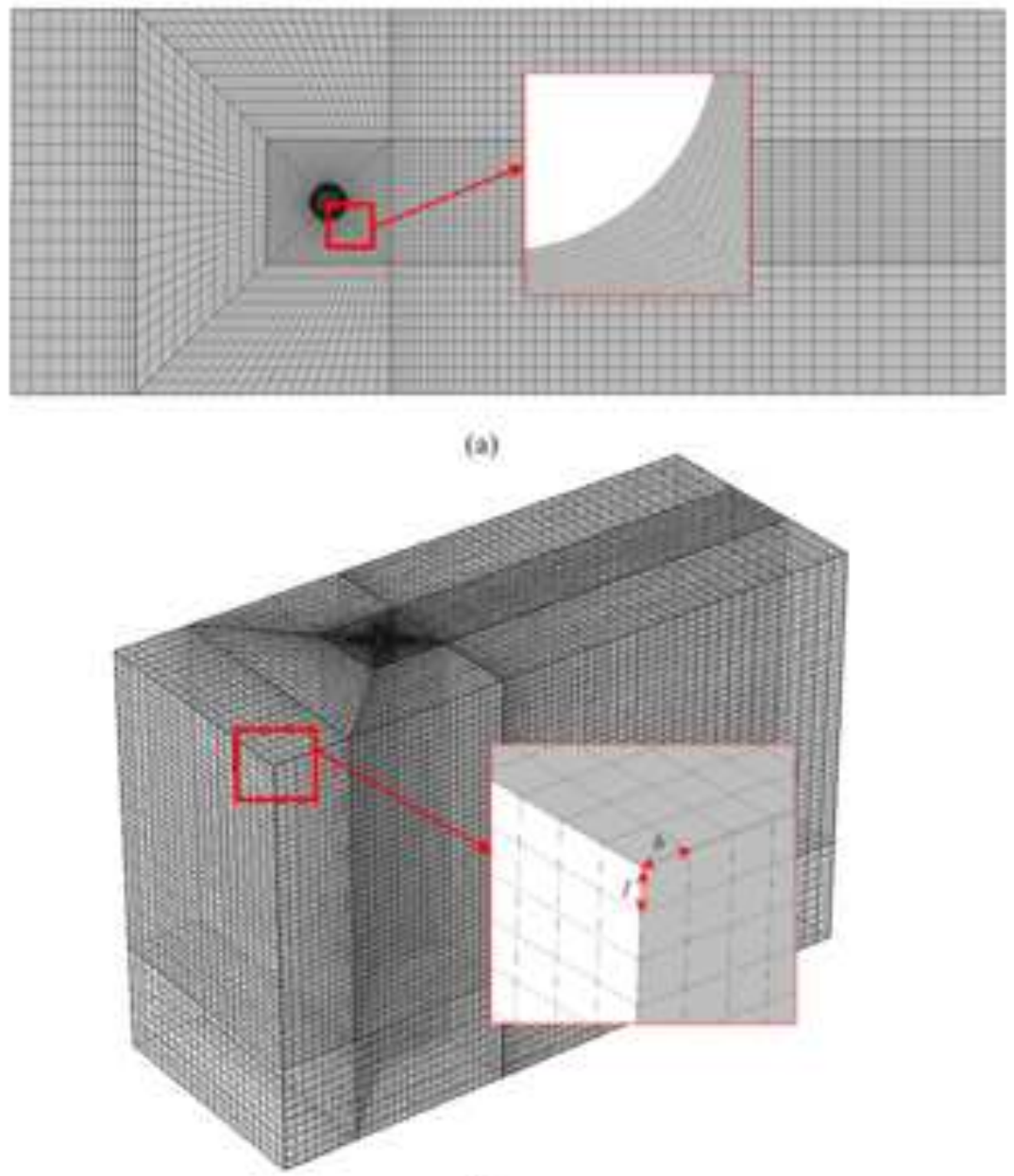

(b) 
Figure 6
Click here to download high resolution image
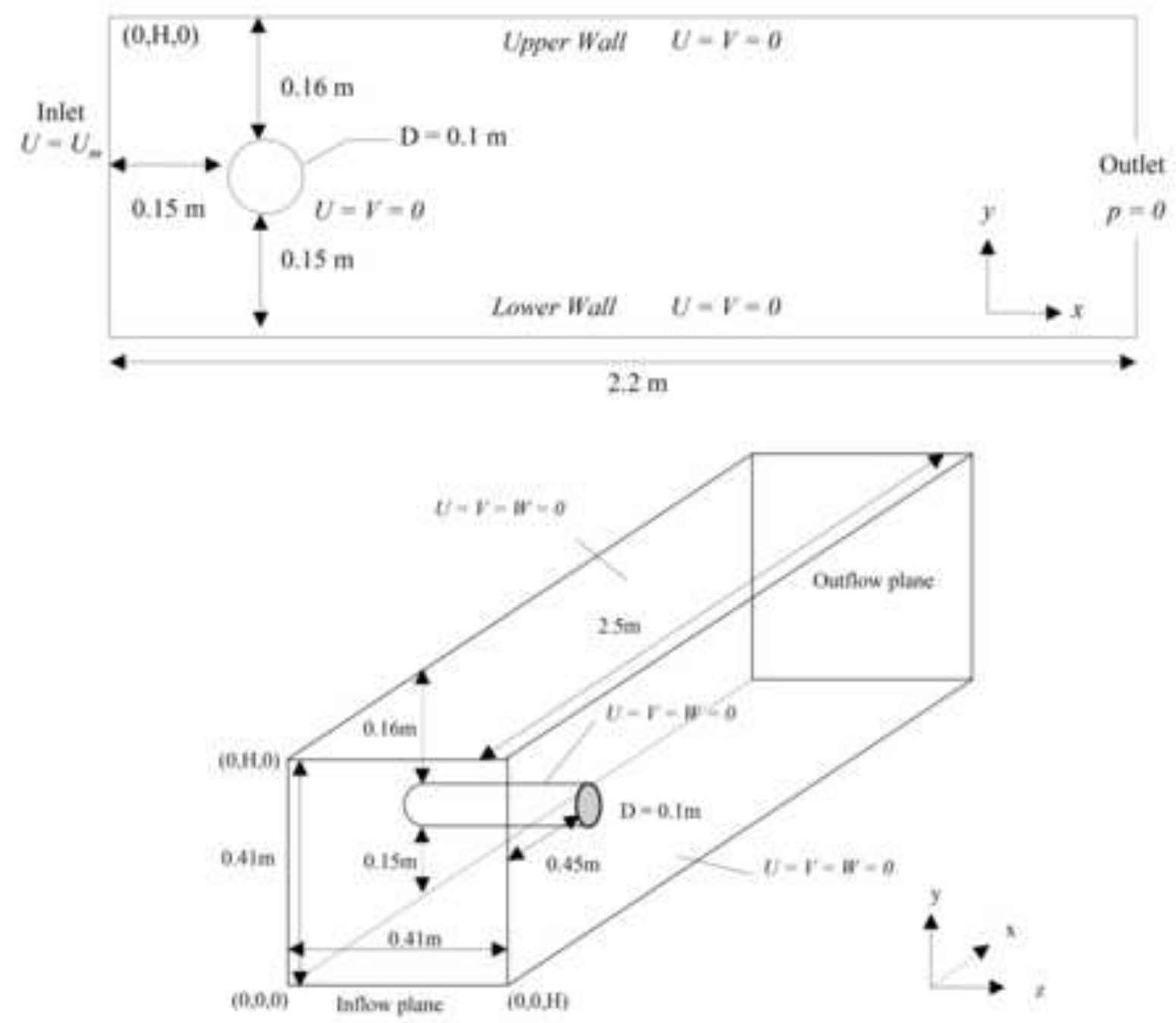
Figure 7
Click here to download high resolution image
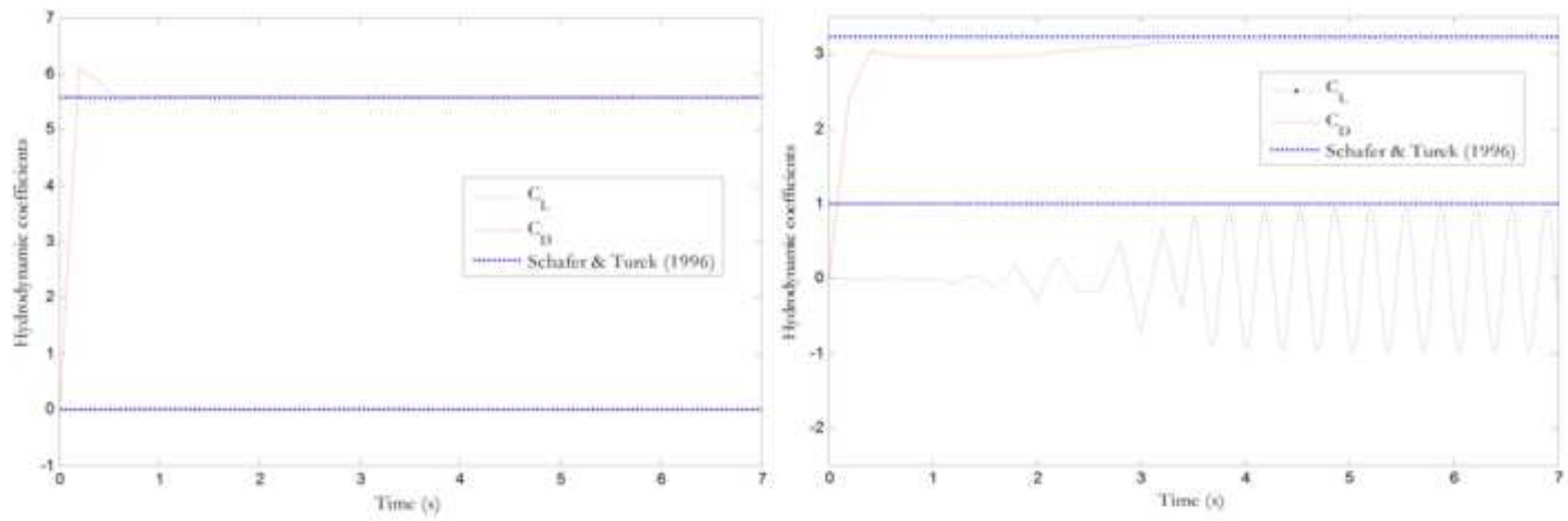
Figure 8
Click here to download high resolution image
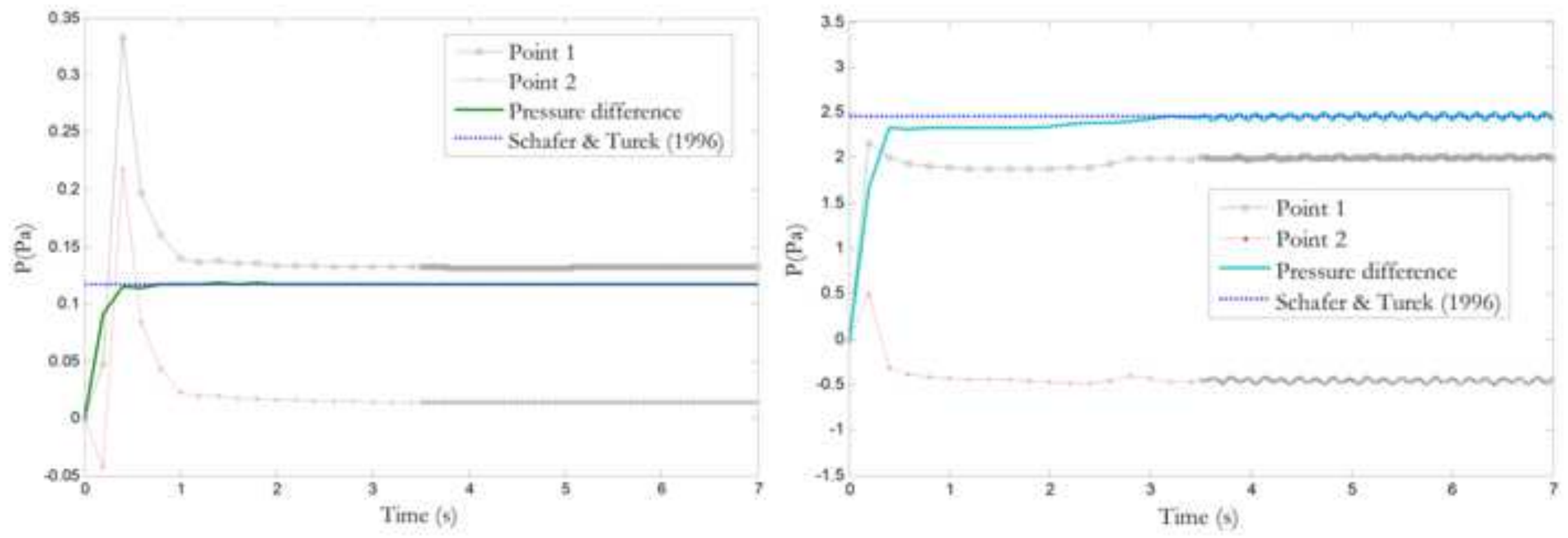
Click here to download high resolution image

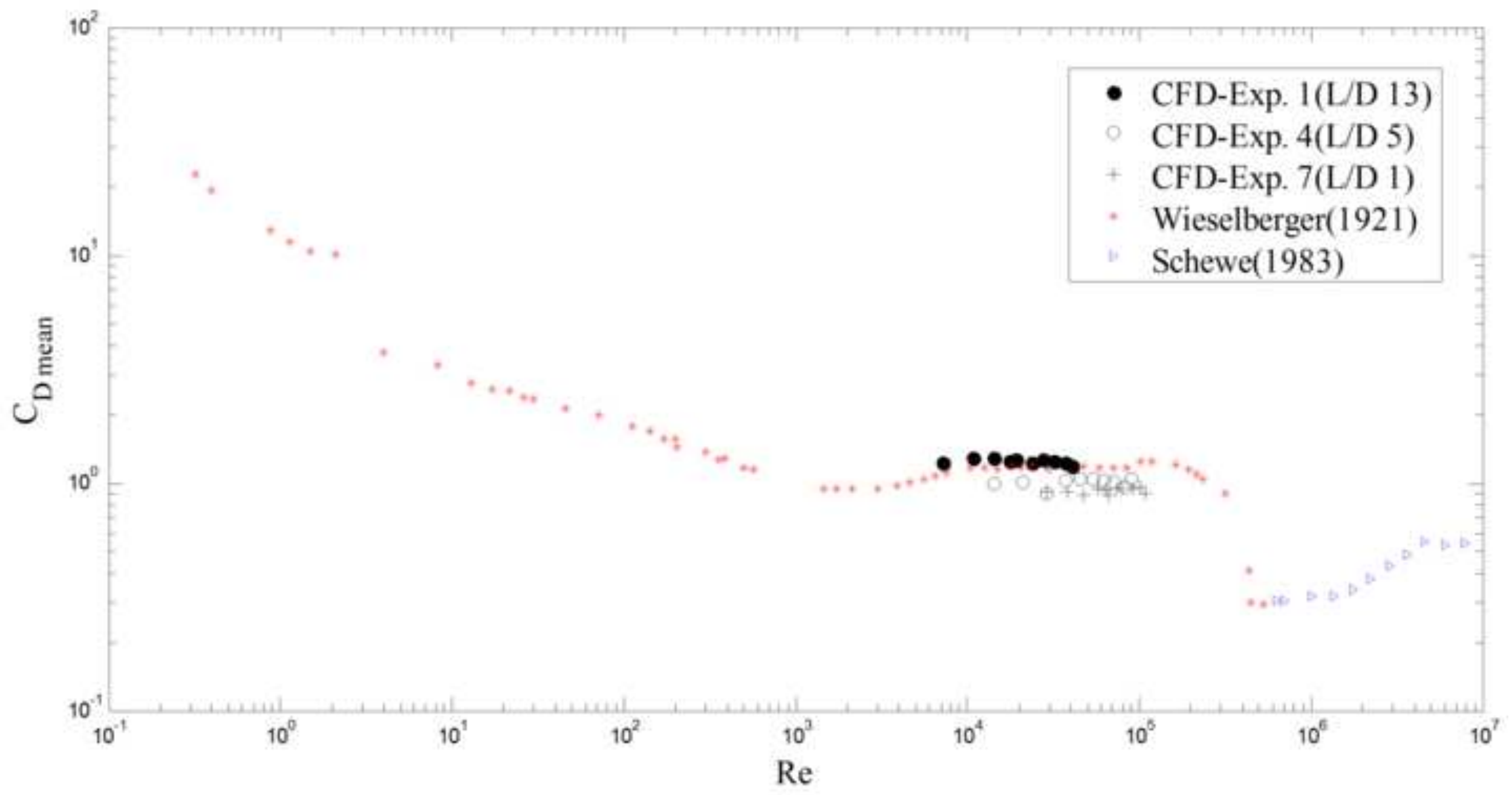


Click here to download high resolution image

(a)

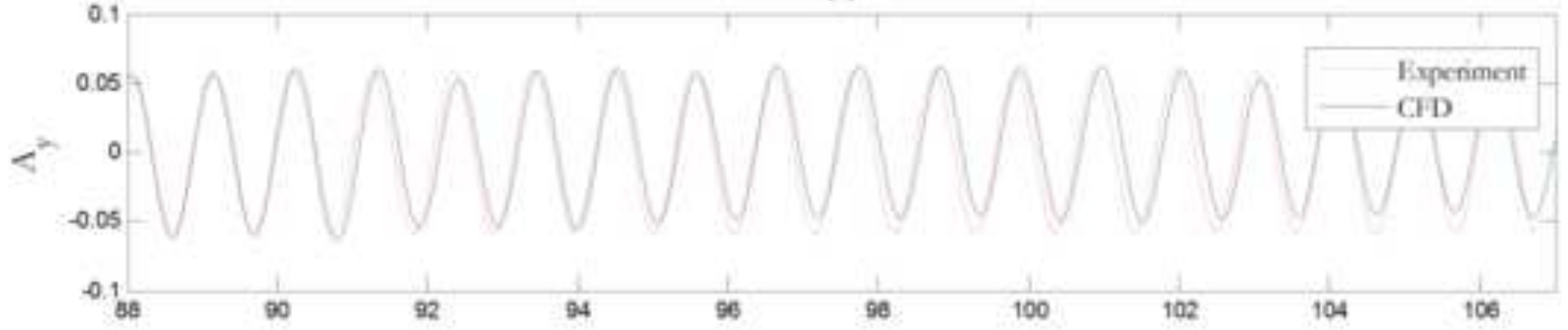

(b)

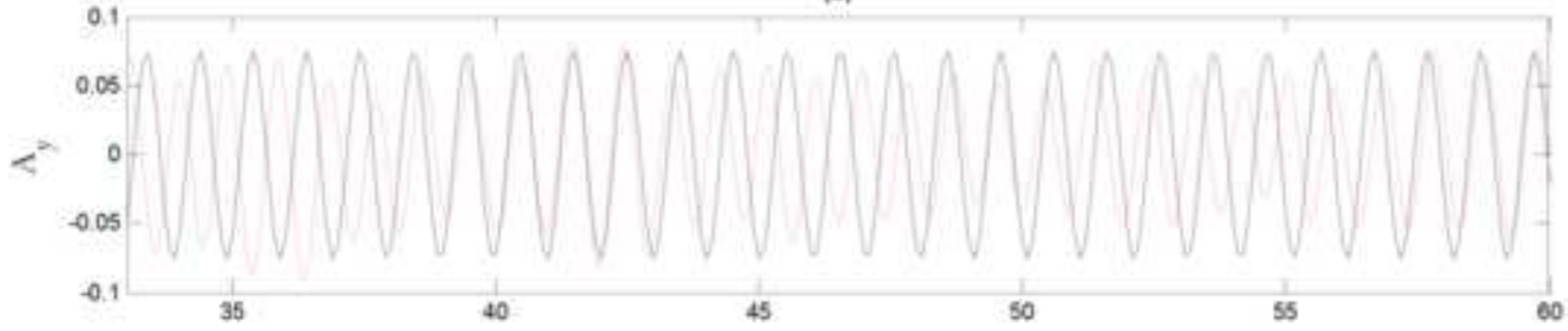

(c)

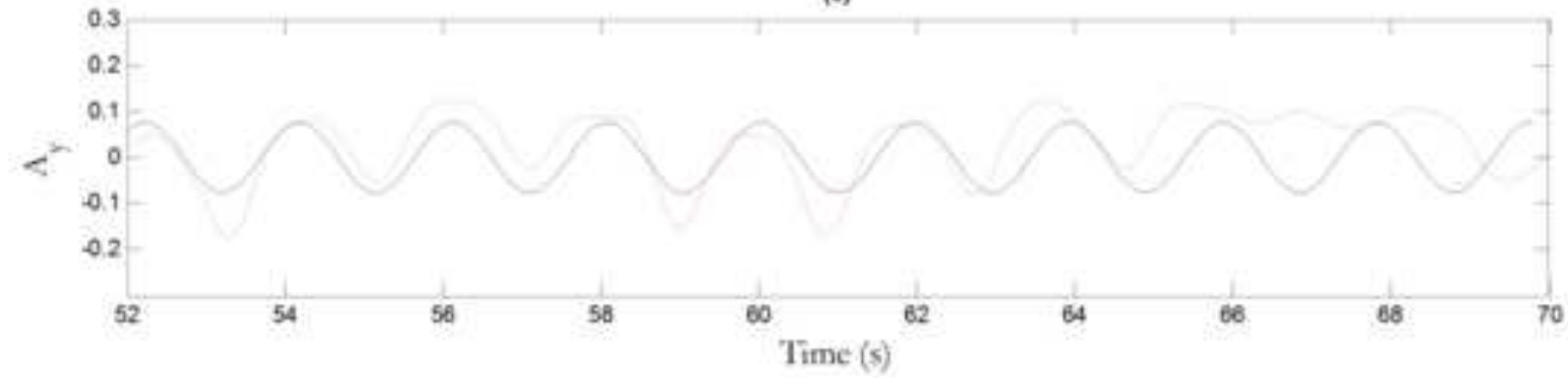


Click here to download high resolution image

(a)

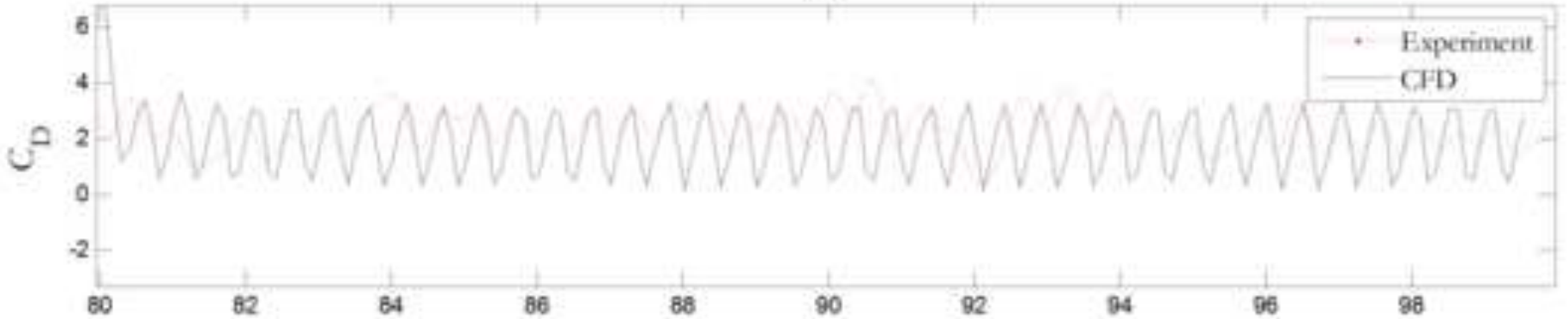

(b)

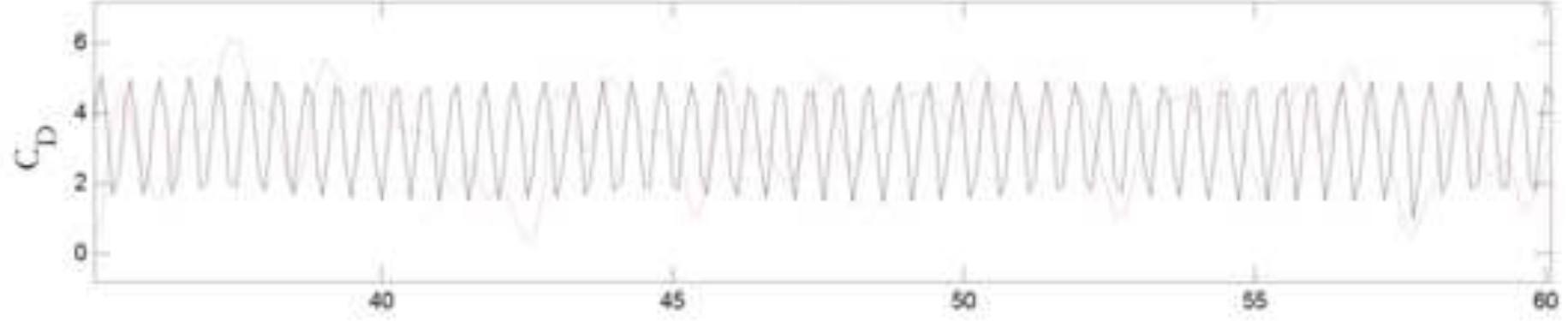

(c)

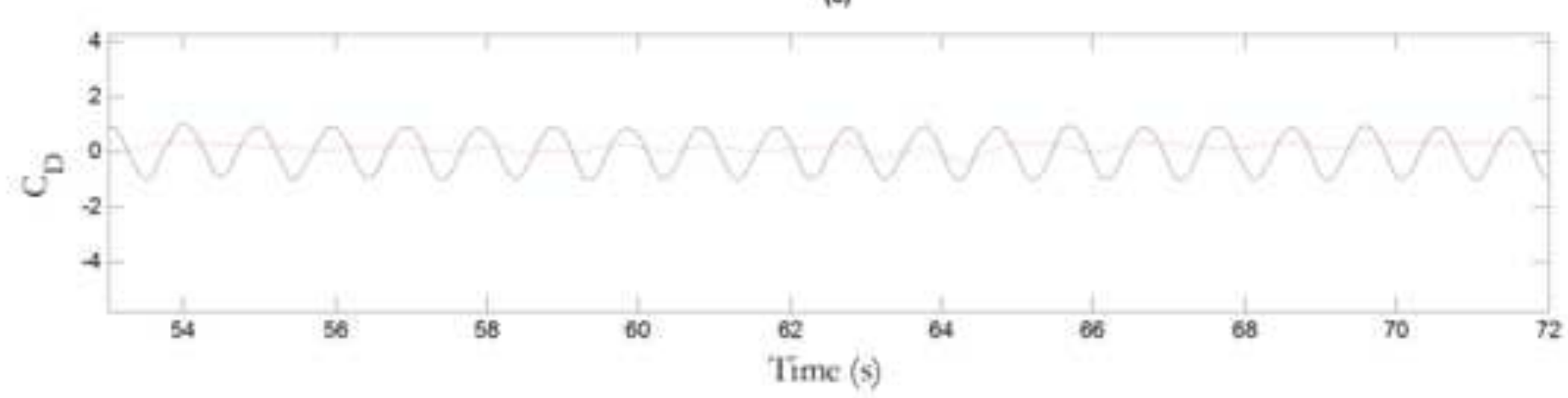

45 60 
Figure 13

Click here to download high resolution image

(a)

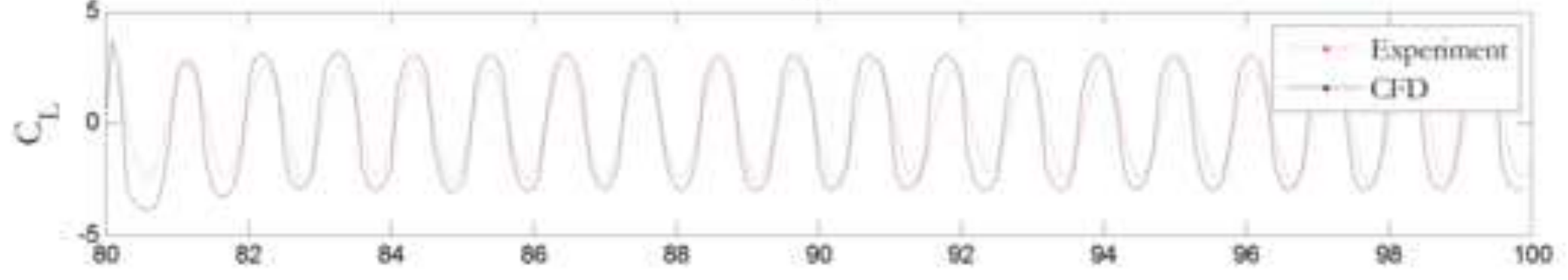

(b)

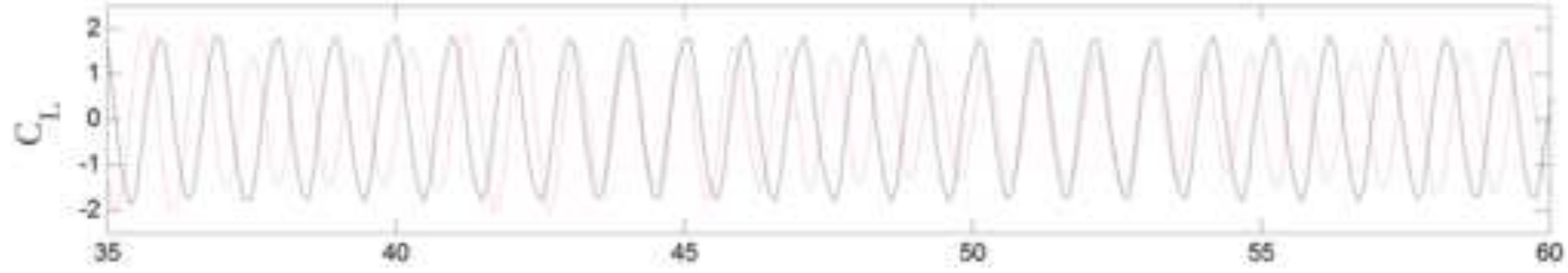

(c)

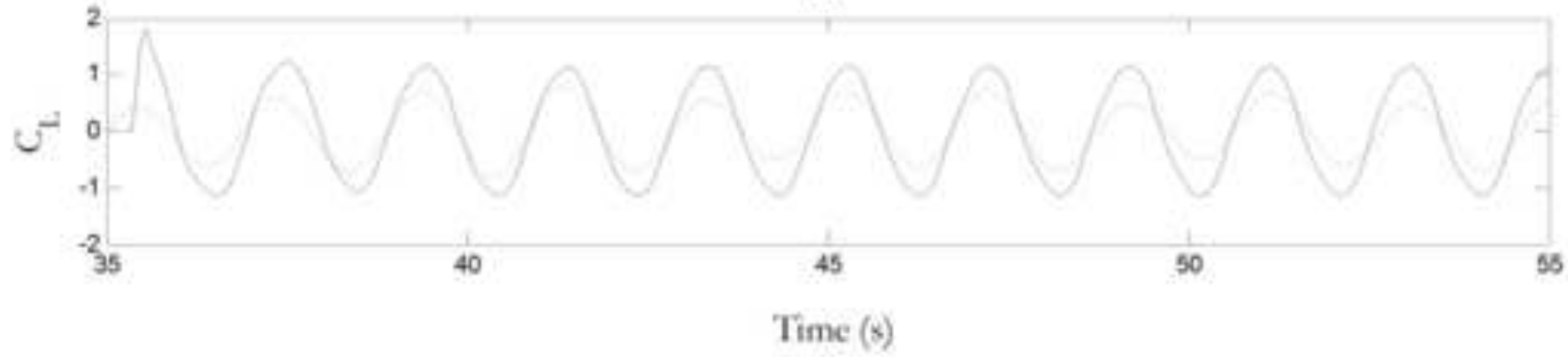

Time (s) 
Click here to download high resolution image

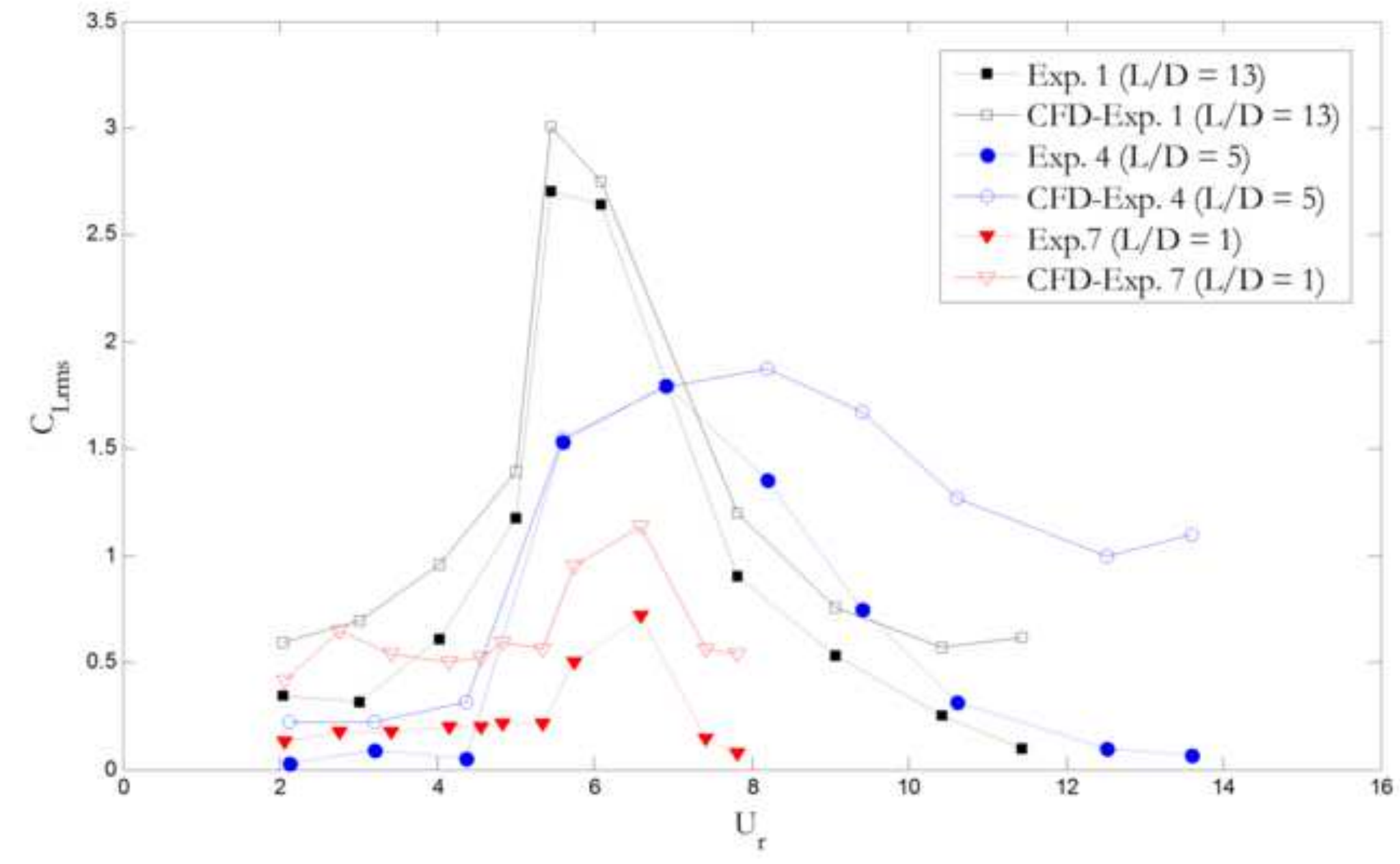




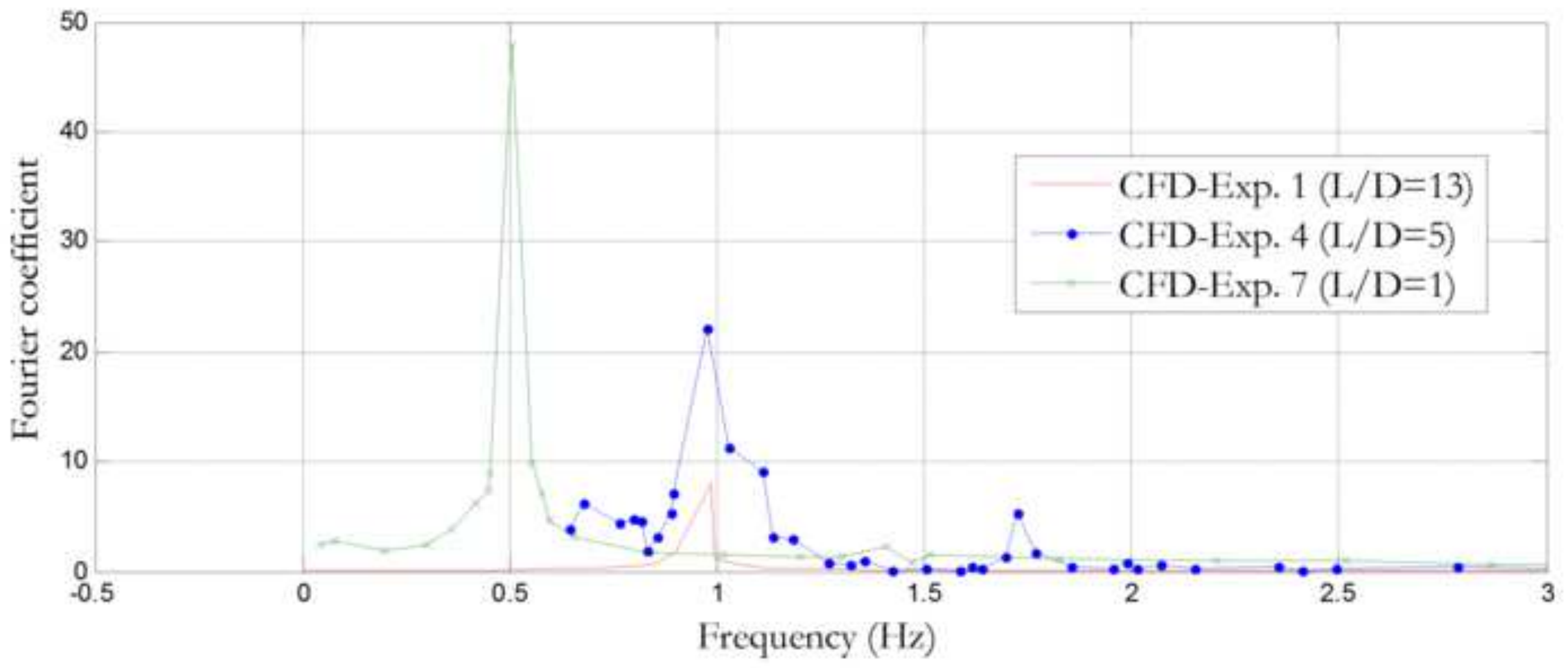


Click here to download high resolution image

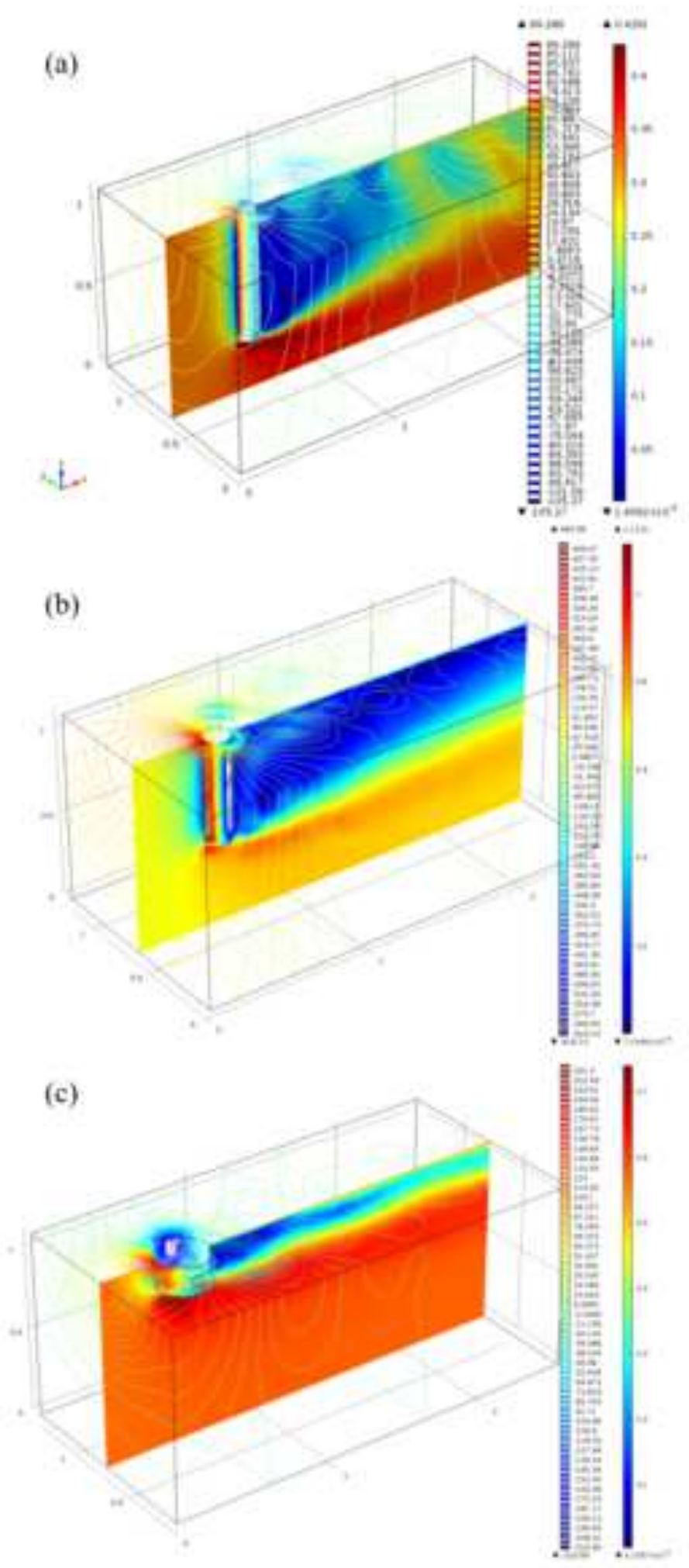


Figure 17
Click here to download high resolution image
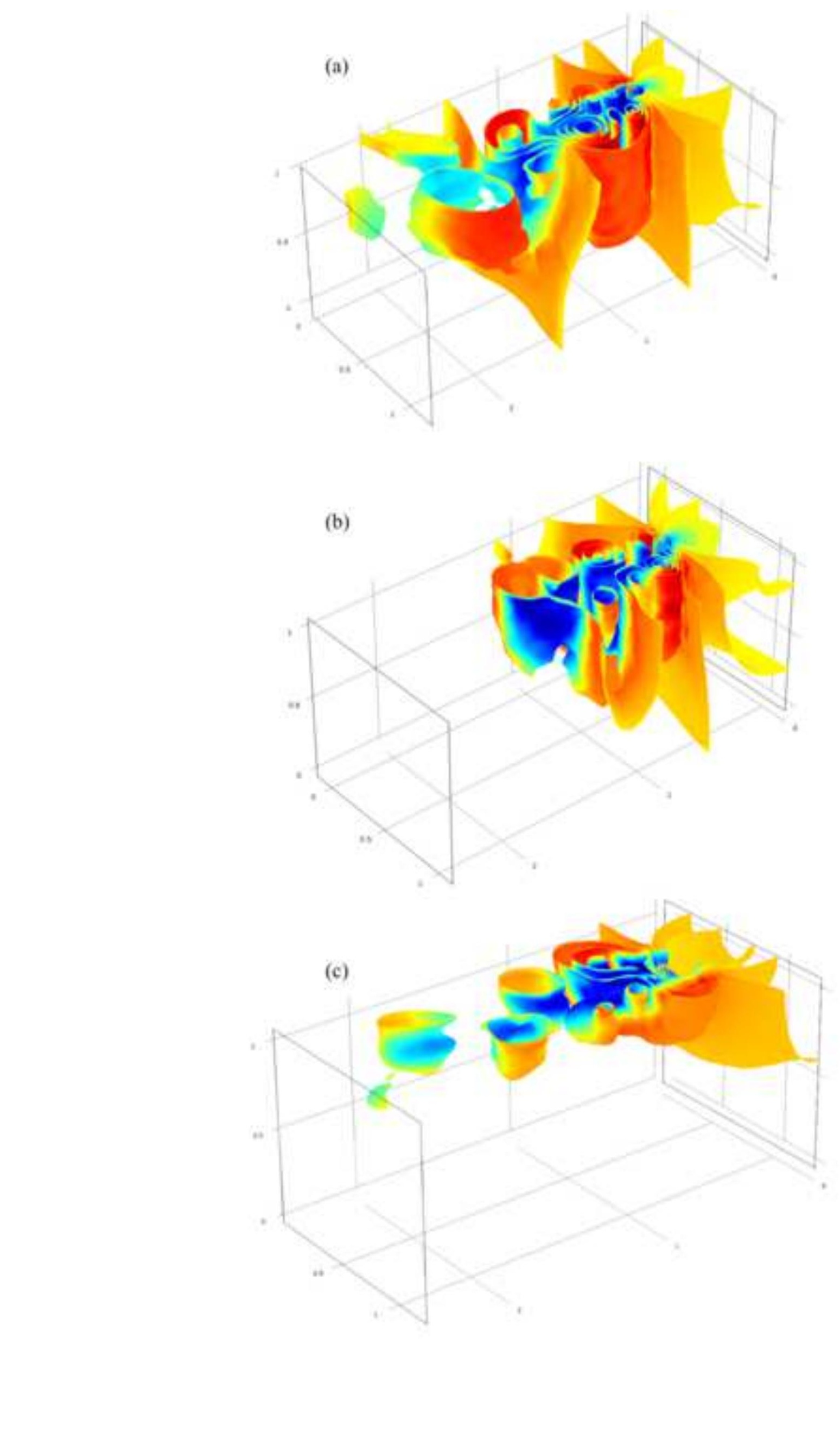

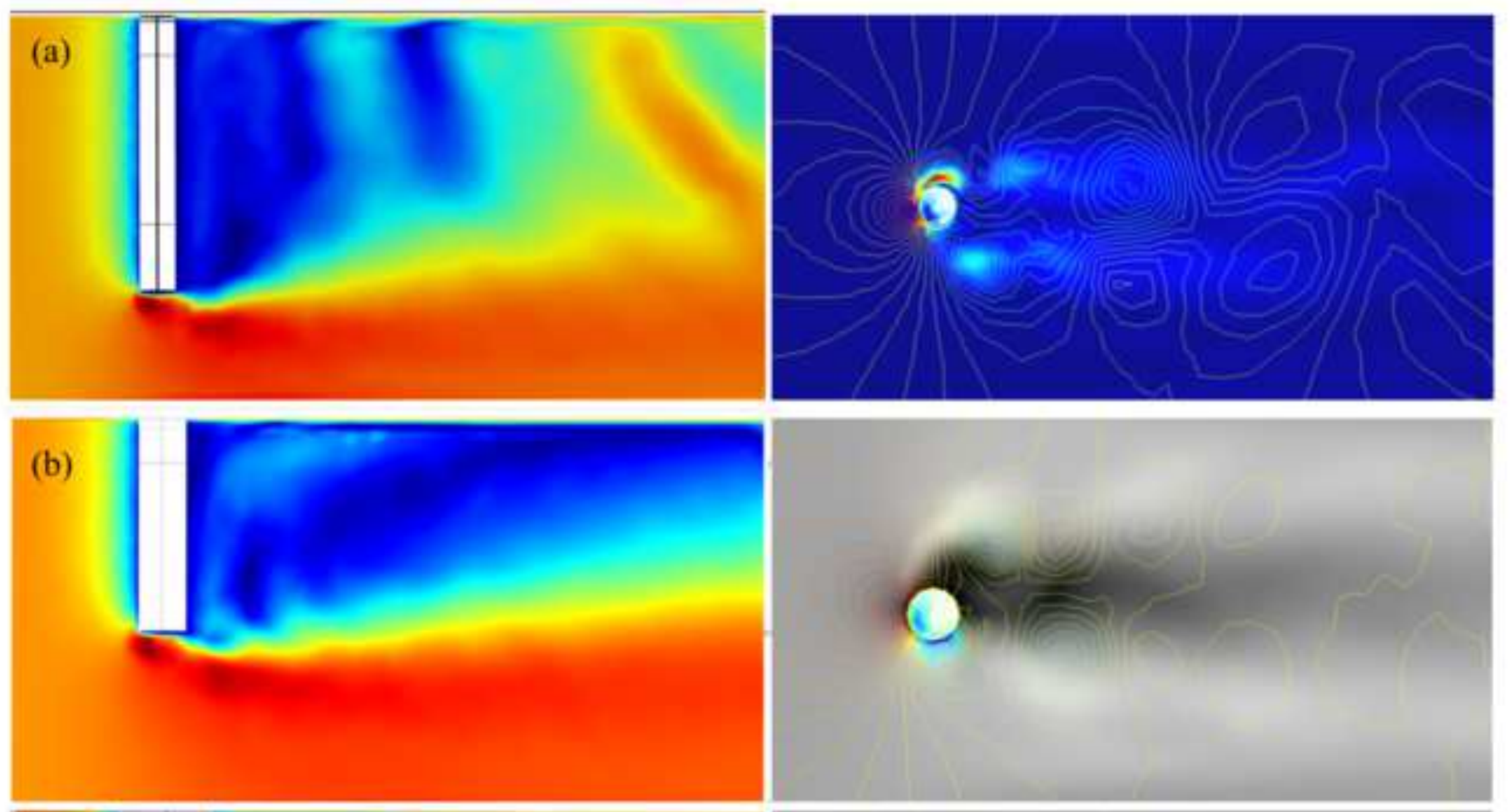

(c)
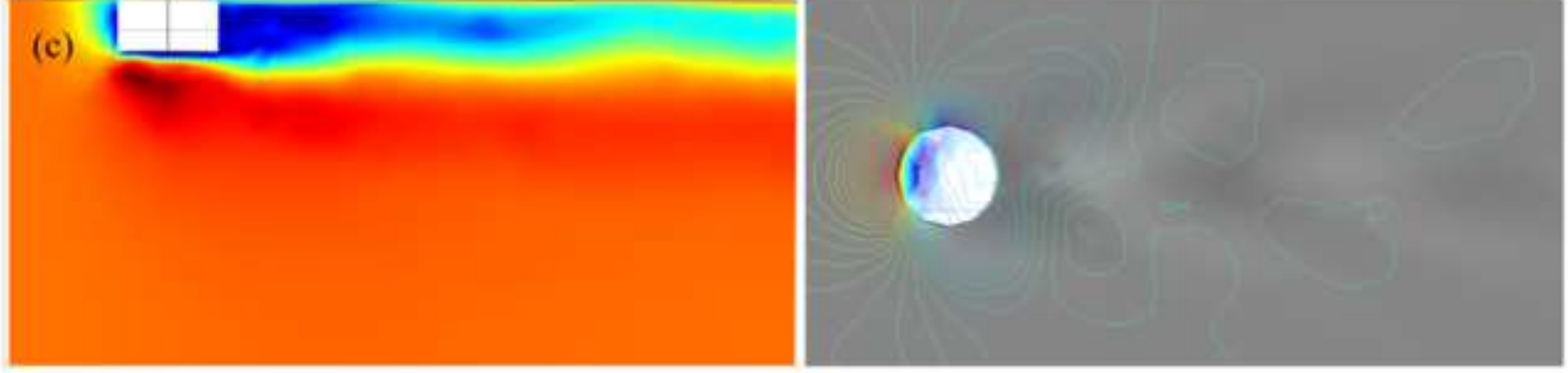